\title{
DISCRETIZATION AND TRANSFERENCE OF BISUBLINEAR MAXIMAL OPERATORS
}

\author{
EARL BERKSON, OSCAR BLASCO, MARÍA J. CARRO, AND T.A. GILLESPIE
}

\begin{abstract}
We develop a general condition for automatically discretizing strong type bisublinear maximal estimates that arise in the context of the real line. In particular, this method applies directly to Michael Lacey's strong type boundedness results for the bisublinear maximal Hilbert transform and for the bisublinear Hardy-Littlewood maximal operator, furnishing the counterpart of each of these two results (without changes to the range of exponents) for the sequence spaces $\ell^{p}(\mathbb{Z})$. We then take up some transference applications of discretized maximal bisublinear operators to maximal estimates and almost everywhere convergence in Lebesgue spaces of abstract measures. We also broaden the scope of such applications, which are based on transference from $\mathbb{Z}$, by developing general methods for transplanting bisublinear maximal estimates from arbitrary locally compact abelian groups.
\end{abstract}

\section{Introduction}

In [10] and [11] R. R. Coifman and G. Weiss expanded the scope of A.P. Calderón's seminal techniques in [9] by developing a wide framework for transferring operators, along with their bounds, from groups to the setting of measure spaces. The resulting "transference" methodology has been developed and its scope extended by many authors, providing a versatile overview for systematically expanding the role of harmonic analysis throughout modern analysis, including abstract Banach spaces. In particular, the transference of individual multilinear operators to measure spaces was introduced in [17], and recently, in [7], the general transference of individual bilinear operators was further developed, branching out to discretization techniques and to transference applications of the Lacey-Thiele result $([27],[28])$ that established the boundedness of the bilinear Hilbert transform for the real line.

The theme of our considerations below will be the transference of strong type bisublinear maximal estimates and their consequent applications to almost everywhere convergence. We start off by developing in Theorem 1.1 below a sufficient condition for the automatic discretization of bisublinear maximal estimates which arise in the context of the real line $\mathbb{R}$. We then show how discrete bisublinear maximal estimates can be transferred to the Lebesgue spaces of abstract measures via isomeries of the Lebesgue spaces-with consequent applications to almost

Date: October, 2005-November, 2005.

2000 Mathematics Subject Classification. Primary 37A05, 42A50, 42B20, 42B25.

Key words and phrases. bilinear, maximal operator, Hardy-Littlewood, Hilbert transform, discretization, transference, measure space, ergodic theory, recurrence.

The second author was partially supported by CICYT BFM2002-0416. The third author was partially supported by MTM2004-02299 and CURE 2001SGR 00069. 
everywhere convergence such as the almost everywhere convergence of the discrete bilinear ergodic Hilbert averages defined by any translation operator in the setting of an arbitrary locally compact abelian group (Theorem 5.1). We thereafter use separation-preserving operators to go beyond the discrete context by developing general methods for transferring bisublinear maximal estimates from arbitrary locally compact abelian groups to Lebesgue spaces of abstract sigma-finite measures.

Our central discretization result in Theorem 1.1 below yields, in particular, the counterparts for the sequence spaces $\ell^{p}(\mathbb{Z})$ of Michael Lacey's strong type boundedness results in [26] for the bisublinear maximal Hilbert transform and for the bisublinear Hardy-Littlewood maximal operator. The resultant discrete bisublinear maximal Hilbert transform and discrete bisublinear Hardy-Littlewood maximal operator (described in Theorems 1.4 and 1.5) then serve as concrete models covered by our transference results. While we shall indicate the role of the discrete bisublinear Hardy-Littlewood maximal operator in such matters, our emphasis will be on the discrete bisublinear maximal Hilbert transform, inasmuch as the recent article [14] is devoted to multisublinear versions of the Hardy-Littlewood maximal operator, including their discretization and transference by measure-preserving transformations to discrete dynamical systems.

For convenience in formulating the central discretization result in Theorem 1.1, we now take up a few items of notation.

Definition 1.1. For $K \in L^{1}(\mathbb{R})$, we shall denote by $S_{K}$ the bilinear mapping of $L^{2}(\mathbb{R}) \times L^{2}(\mathbb{R})$ into $L^{1}(\mathbb{R})$ specified by

$$
\left(S_{K}(f, g)\right)(x)=\int_{\mathbb{R}} f(x+y) g(x-y) K(y) d y
$$

Given a sequence $\left\{K_{j}\right\}_{j=1}^{\infty} \subseteq L^{1}(\mathbb{R})$, the corresponding bisublinear maximal operator will be symbolized by $S_{\left\{K_{j}\right\}}^{b}$. Thus, for $f, g \in L^{2}(\mathbb{R})$, and almost all $x \in \mathbb{R}$,

$$
\left(S_{\left\{K_{j}\right\}}^{b}(f, g)\right)(x)=\sup _{j \in \mathbb{N}}\left|\left(S_{K_{j}}(f, g)\right)(x)\right| .
$$

Definition 1.2. Let $\mathcal{K}=\left\{\mathcal{K}_{n}\right\}_{n=-\infty}^{\infty}$ be a sequence of complex numbers belonging to $\ell^{1}(\mathbb{Z})$, and suppose that $0<p_{1, p_{2}} \leq \infty$. Since $\ell^{\infty}(\mathbb{Z})$ contains $\ell^{p_{1}}(\mathbb{Z}) \cup \ell^{p_{2}}(\mathbb{Z})$, it is clear that for all $a \in \ell^{p_{1}}$, all $b \in \ell^{p_{2}}(\mathbb{Z})$, and all $m \in \mathbb{Z}$,

$$
\sum_{n=-\infty}^{\infty}\left|a_{m+n} b_{m-n} \mathcal{K}_{n}\right|<\infty
$$

and we shall denote by $\mathfrak{S}_{\mathcal{K}}$ the bilinear mapping which takes $\ell^{p_{1}}(\mathbb{Z}) \times \ell^{p_{2}}(\mathbb{Z})$ into the complex-valued sequences defined on $\mathbb{Z}$, and which is specified for all $a \in \ell^{p_{1}}$, all $b \in \ell^{p_{2}}(\mathbb{Z})$, and all $m \in \mathbb{Z}$ by putting

$$
\left(\mathfrak{S}_{\mathcal{K}}(a, b)\right)(m)=\sum_{n=-\infty}^{\infty} a_{m+n} b_{m-n} \mathcal{K}_{n}
$$

Given a sequence $\left\{\mathcal{K}^{(j)}\right\}_{j=1}^{\infty}$ of elements of $\ell^{1}(\mathbb{Z})$, the corresponding bisublinear maximal operator will be symbolized by $\mathfrak{S}_{\left\{\mathcal{K}^{(j)}\right\}}^{b}$, and so for $a \in \ell^{p_{1}}, b \in \ell^{p_{2}}(\mathbb{Z})$, 
and $m \in \mathbb{Z}$,

$$
\left(\mathfrak{S}_{\left\{\mathcal{K}^{(j)}\right\}}^{b}(a, b)\right)(m)=\sup _{j \in \mathbb{N}}\left|\left(\mathfrak{S}_{\mathcal{K}^{(j)}}(a, b)\right)(m)\right| .
$$

The linear space of all complex-valued bilateral sequences which have finite support will be denoted by $\ell_{0}(\mathbb{Z})$. Notice that if $\mathcal{K} \in \ell^{1}(\mathbb{Z})$, then $\mathfrak{S}_{\mathcal{K}}$ maps $\ell_{0}(\mathbb{Z}) \times \ell_{0}(\mathbb{Z})$ into $\ell_{0}(\mathbb{Z})$. Throughout all that follows, let $\mathcal{I}$ designate the closed interval $\left[-\frac{1}{4}, \frac{1}{4}\right]$ in $\mathbb{R}$, and for each $m \in \mathbb{Z}$, let $\mathcal{I}_{m}=\mathcal{I}+m$.

Remark 1.1. Suppose that $K \in L^{1}(\mathbb{R})$, and that for each $n \in \mathbb{Z}$, the restriction $K \mid \mathcal{I}_{n}$ has a continuous derivative (in symbols, $\left(K \mid \mathcal{I}_{n}\right) \in C^{1}\left(\mathcal{I}_{n}\right)$ ). Put

$$
\left\|\left(K \mid \mathcal{I}_{n}\right)^{\prime}\right\|_{u}=\sup _{x \in \mathcal{I}_{n}}\left|\left(K \mid \mathcal{I}_{n}\right)^{\prime}(x)\right| \text {. }
$$

The following elementary argument shows that if $\left\{\left\|\left(K \mid \mathcal{I}_{n}\right)^{\prime}\right\|_{u}\right\} \in \ell^{1}(\mathbb{Z})$, then the sequence $\mathcal{K} \equiv\{K(n)\}_{n=-\infty}^{\infty} \in \ell^{1}(\mathbb{Z})$ (and hence the bilinear form $S_{K}$ specified in (1.1) can be discretized to the bilinear form $\mathfrak{S}_{\mathcal{K}}$ given by (1.3)). For each $n \in \mathbb{Z}$,

$$
\frac{|K(n)|}{2} \leq \int_{\mathcal{I}_{n}}|K(n)-K(y)| d y+\int_{\mathcal{I}_{n}}|K(y)| d y,
$$

and since for each $y \in \mathcal{I}_{n}$,

$$
|K(y)-K(n)|=\left|\int_{n}^{y} K^{\prime}(t) d t\right| \leq\left\|\left(K \mid \mathcal{I}_{n}\right)^{\prime}\right\|_{u}|y-n|,
$$

we have

$$
\begin{aligned}
\frac{|K(n)|}{2} & \leq\left\|\left(K \mid \mathcal{I}_{n}\right)^{\prime}\right\|_{u} \int_{\mathcal{I}_{n}}|y-n| d y+\int_{\mathcal{I}_{n}}|K(y)| d y \\
& =\frac{\left\|\left(K \mid \mathcal{I}_{n}\right)^{\prime}\right\|_{u}}{16}+\int_{\mathcal{I}_{n}}|K(y)| d y .
\end{aligned}
$$

We can now state our fundamental discretization theorem (whose demonstration will be deferred to $\S 2$ ).

Theorem 1.1. Suppose that

$$
\begin{gathered}
0<p_{1}, p_{2}, p_{3} \leq \infty ; \\
\frac{1}{p_{1}}+\frac{1}{p_{2}}=\frac{1}{p_{3}} .
\end{gathered}
$$

Let $\left\{K_{j}\right\}_{j=1}^{\infty}$ be a sequence of functions belonging to $L^{1}(\mathbb{R})$ such that for some constant $\mathfrak{C}$ (depending on $p_{1}, p_{2}$, and $\left\{K_{j}\right\}_{j=1}^{\infty}$ ) we have

$$
\begin{aligned}
& \left\|S_{\left\{K_{j}\right\}}^{b}(f, g)\right\|_{L^{p_{3}(\mathbb{R})}} \leq \mathfrak{C}\|f\|_{L^{p_{1}(\mathbb{R})}}\|g\|_{L^{p_{2}(\mathbb{R})}}, \\
& \quad \text { for all } f \in L^{p_{1}}(\mathbb{R}) \bigcap L^{2}(\mathbb{R}), \text { all } g \in L^{p_{2}}(\mathbb{R}) \bigcap L^{2}(\mathbb{R}) .
\end{aligned}
$$

Assume that for each $j \in \mathbb{N}$, the restriction $\left(K_{j} \mid \mathcal{I}_{n}\right)$ belongs to $C^{1}\left(\mathcal{I}_{n}\right)$ for each $n \in \mathbb{Z}$, and define

by putting

$$
A_{n}=A_{n}\left(\left\{K_{j}\right\}_{j=1}^{\infty}\right)
$$

$$
A_{n}=\sup \left\{\left|\left(K_{j} \mid \mathcal{I}_{n}\right)^{\prime}(x)\right|: j \in \mathbb{N}, x \in \mathcal{I}_{n}\right\} .
$$


Let $p^{*}=\min \left(p_{3}, 1\right)$, and suppose that

$$
\left\{A_{n}\right\}_{n=-\infty}^{\infty} \in \ell^{p^{*}}(\mathbb{Z}) .
$$

Then the following conclusions hold.

(i) For each $j \in \mathbb{N}, \ell^{1}(\mathbb{Z})$ contains the sequence of complex numbers $\mathcal{K}^{(j)}$ $\equiv\left\{\mathcal{K}_{n}^{(j)}\right\}_{n=-\infty}^{\infty}$ specified by taking

$$
\mathcal{K}_{n}^{(j)}=K_{j}(n), \text { for all } n \in \mathbb{Z} .
$$

(ii) The discrete bisublinear maximal operator $\mathfrak{S}_{\left\{\mathcal{K}^{(j)}\right\}}^{b}$ specified by (1.4) satisfies a strong type estimate

$$
\begin{array}{r}
\left\|\mathfrak{S}_{\left\{\mathcal{K}^{(j)}\right\}}^{b}(a, b)\right\|_{\ell^{p_{3}}(\mathbb{Z})} \leq \eta\|a\|_{\ell^{p_{1}}(\mathbb{Z})}\|b\|_{\ell^{p_{2}}(\mathbb{Z})}, \\
\text { for all } a \in \ell^{p_{1}}(\mathbb{Z}), \text { and all } b \in \ell^{p_{2}}(\mathbb{Z}),
\end{array}
$$

where $\eta$ is a constant depending only on $p_{1}, p_{2}$, and $\left\{K_{j}\right\}_{j=1}^{\infty}$.

As will be discussed shortly, Theorem 1.1 readily furnishes discrete counterparts to the following two boundedness results of Michael Lacey ([26]) for the bisublinear maximal Hilbert transform and for the bisublinear Hardy-Littlewood maximal operator.

Theorem 1.2. Suppose that

$$
\begin{gathered}
1<p_{1}, p_{2} \leq \infty \\
\frac{1}{p_{1}}+\frac{1}{p_{2}}=\frac{1}{p_{3}} \\
\frac{2}{3}<p_{3}<\infty
\end{gathered}
$$

For $f \in L^{p_{1}}(\mathbb{R}) \bigcap L^{2}(\mathbb{R}), g \in L^{p_{2}}(\mathbb{R}) \bigcap L^{2}(\mathbb{R})$, let

$$
\mathcal{H}(f, g)(x)=\sup _{0<\varepsilon<\delta<\infty}\left|\int_{\varepsilon<|y|<\delta} \frac{f(x+y) g(x-y)}{y} d y\right| \text {, for all } x \in \mathbb{R} .
$$

Then there is a constant $A_{p_{1}, p_{2}}$, depending only on $p_{1}$ and $p_{2}$, such that

$$
\begin{aligned}
& \|\mathcal{H}(f, g)\|_{L^{p_{3}(\mathbb{R})}} \leq A_{p_{1}, p_{2}}\|f\|_{L^{p_{1}(\mathbb{R})}}\|g\|_{L^{p_{2}(\mathbb{R})}}, \\
& \quad \text { for all } f \in L^{p_{1}}(\mathbb{R}) \bigcap L^{2}(\mathbb{R}), g \in L^{p_{2}}(\mathbb{R}) \bigcap L^{2}(\mathbb{R}) .
\end{aligned}
$$

Theorem 1.3. Suppose that

$$
\begin{gathered}
1<p_{1}, p_{2}<\infty ; \\
\frac{1}{p_{1}}+\frac{1}{p_{2}}=\frac{1}{p_{3}}<\frac{3}{2} .
\end{gathered}
$$

For $f \in L^{p_{1}}(\mathbb{R}) \bigcap L^{2}(\mathbb{R}), g \in L^{p_{2}}(\mathbb{R}) \bigcap L^{2}(\mathbb{R})$, let

$$
\mathcal{M}(f, g)(x)=\sup _{t>0} \frac{1}{2 t} \int_{-t}^{t}|f(x+y) g(x-y)| d y, \text { for all } x \in \mathbb{R} .
$$

Then there is a constant $B_{p_{1}, p_{2}}$, depending only on $p_{1}$ and $p_{2}$, such that

$$
\begin{aligned}
& \|\mathcal{M}(f, g)\|_{L^{p_{3}(\mathbb{R})}} \leq B_{p_{1}, p_{2}}\|f\|_{L^{p_{1}(\mathbb{R})}}\|g\|_{L^{p_{2}(\mathbb{R})}}, \\
& \quad \text { for all } f \in L^{p_{1}}(\mathbb{R}) \bigcap L^{2}(\mathbb{R}), g \in L^{p_{2}}(\mathbb{R}) \bigcap L^{2}(\mathbb{R}) .
\end{aligned}
$$


The discretized versions of the above two theorems are stated as follows.

Theorem 1.4. Suppose that the conditions (1.12), (1.13), and (1.14) hold. For $a \equiv\left\{a_{n}\right\}_{n=-\infty}^{\infty} \in \ell^{p_{1}}(\mathbb{Z}), b \equiv\left\{b_{n}\right\}_{n=-\infty}^{\infty} \in \ell^{p_{2}}(\mathbb{Z})$, let

$$
\mathcal{H}_{\mathbb{Z}}(a, b)(m)=\sup _{j \in \mathbb{N}}\left|\sum_{0<|n| \leq j} \frac{a_{m+n} b_{m-n}}{n}\right|, \text { for all } m \in \mathbb{Z} .
$$

Then there is a constant $\mathfrak{A}_{p_{1}, p_{2}}$, depending only on $p_{1}$ and $p_{2}$, such that

$$
\begin{array}{r}
\left\|\mathcal{H}_{\mathbb{Z}}(a, b)\right\|_{\ell^{p_{3}(\mathbb{Z})}} \leq \mathfrak{A}_{p_{1}, p_{2}}\|a\|_{\ell^{p_{1}}(\mathbb{Z})}\|b\|_{\ell^{p_{2}}(\mathbb{Z})}, \\
\text { for all } a \in \ell^{p_{1}}(\mathbb{Z}), b \in \ell^{p_{2}}(\mathbb{Z}) .
\end{array}
$$

Theorem 1.5. Suppose that the conditions (1.17) and (1.18) hold. For $a \equiv$ $\left\{a_{n}\right\}_{n=-\infty}^{\infty} \in \ell^{p_{1}}(\mathbb{Z}), b \equiv\left\{b_{n}\right\}_{n=-\infty}^{\infty} \in \ell^{p_{2}}(\mathbb{Z})$, let

$$
\mathcal{M}_{\mathbb{Z}}(a, b)(m)=\sup _{j \in \mathbb{N}} \frac{1}{2 j+1} \sum_{n=-j}^{j}\left|a_{m+n} b_{m-n}\right|, \text { for all } m \in \mathbb{Z} .
$$

Then there is a constant $\mathfrak{B}_{p_{1}, p_{2}}$, depending only on $p_{1}$ and $p_{2}$, such that

$$
\begin{gathered}
\left\|\mathcal{M}_{\mathbb{Z}}(a, b)\right\|_{\ell^{p_{3}}(\mathbb{Z})} \leq \mathfrak{B}_{p_{1}, p_{2}}\|a\|_{\ell^{p_{1}}(\mathbb{Z})}\|b\|_{\ell^{p_{2}(\mathbb{Z})}}, \\
\text { for all } a \in \ell^{p_{1}}(\mathbb{Z}), b \in \ell^{p_{2}}(\mathbb{Z}) .
\end{gathered}
$$

To deduce Theorem 1.4 and Theorem 1.5 from Theorem 1.2 and Theorem 1.3, respectively, we reason via Theorem 1.1 as follows. (Strictly speaking, to complete the proof in the case of $\mathcal{M}_{\mathbb{Z}}$ we shall also need to apply the corresponding outcome of Theorem 1.1 to $|a|$ and $|b|$ ). In the case of Theorem 1.4, for each $j \in \mathbb{N}$, we take $K_{j} \in L^{1}(\mathbb{R})$ to be the function specified for every $x \in \mathbb{R}$ by

$$
K_{j}(x)=\left\{\begin{array}{cc}
x^{-1}, & \text { if } \frac{3}{4} \leq|x| \leq j+\frac{1}{4} \\
0, & \text { otherwise. }
\end{array}\right.
$$

In the case of Theorem 1.5 , we define each $K_{j} \in L^{1}(\mathbb{R})$ by putting

$$
K_{j}(x)=\left\{\begin{array}{cc}
(2 j+1)^{-1}, & \text { if }|x| \leq j+\frac{1}{2} \\
0, & \text { otherwise }
\end{array}\right.
$$

It then suffices to show that (1.10) holds in the each of the present contexts. This outcome occurs in the case of $(1.25)$, because $p^{*}>\frac{2}{3}$, while for all $n \in \mathbb{Z} \backslash\{0\}$,

$$
A_{n} \leq \frac{C}{n^{2}}
$$

In the case of $(1.26),(1.10)$ holds trivially, since $A_{n}=0$ for all $n \in \mathbb{Z}$.

Remark 1.2. Because of the positivity of the family of kernels for the bisublinear Hardy-Littlewood maximal operator, a more direct and immediate derivation of Theorem 1.5 from Theorem 1.3 can be carried out (see Proposition 14.1-(iii) of [14]). 
After recourse to a suitable multilinear version of the classical Banach Principle (see the reasoning for either Theorem 1.2.1 in [13] or Theorem 6 in [18], whose statement is reproduced below in Proposition 3.1), one observes directly that the maximal result in Theorem 1.2 subsumes the celebrated Lacey-Thiele boundedness result for the bilinear Hilbert transform of $\mathbb{R}([27]$, [28]), which solved the longstanding Calderón conjecture. We observe here that the corresponding boundedness result for the discrete bilinear Hilbert transform follows even more simply from Theorem 1.4.

Theorem 1.6. Suppose that the conditions (1.12), (1.13), and (1.14) hold. Then for $a \equiv\left\{a_{j}\right\}_{j=-\infty}^{\infty} \in \ell^{p_{1}}(\mathbb{Z}), b \equiv\left\{b_{j}\right\}_{j=-\infty}^{\infty} \in \ell^{p_{2}}(\mathbb{Z})$, the series

$$
\left(H_{\mathbb{Z}}(a, b)\right)(m) \equiv \sum_{\substack{n=-\infty \\ n \neq 0}}^{\infty} \frac{a_{m+n} b_{m-n}}{n}
$$

converges absolutely for each $m \in \mathbb{Z}$, and the corresponding bilinear operator $H_{\mathbb{Z}}$ defined on $\ell^{p_{1}}(\mathbb{Z}) \times \ell^{p_{2}}(\mathbb{Z})$ satisfies

$$
\begin{gathered}
\left\|H_{\mathbb{Z}}(a, b)\right\|_{\ell^{p_{3}}(\mathbb{Z})} \leq \mathfrak{A}_{p_{1}, p_{2}}\|a\|_{\ell^{p_{1}}(\mathbb{Z})}\|b\|_{\ell^{p_{2}}(\mathbb{Z})}, \\
\text { for all } a \in \ell^{p_{1}}(\mathbb{Z}), b \in \ell^{p_{2}}(\mathbb{Z}),
\end{gathered}
$$

where $\mathfrak{A}_{p_{1}, p_{2}}$ is the constant occurring in (1.22). For $a \equiv\left\{a_{j}\right\}_{j=-\infty}^{\infty} \in \ell^{p_{1}}(\mathbb{Z})$, $b \equiv\left\{b_{j}\right\}_{j=-\infty}^{\infty} \in \ell^{p_{2}}(\mathbb{Z})$, and $j \in \mathbb{N}$, let

$$
\begin{gathered}
H_{j, \mathbb{Z}}(a, b)(m)=\sum_{\substack{0<|n| \leq j \\
\text { for all } m \in \mathbb{Z} .}} \frac{a_{m+n} b_{m-n}}{n}, \\
\text { for }
\end{gathered}
$$

Then $\left\{H_{j, \mathbb{Z}}(a, b)\right\}_{j=1}^{\infty}$ converges to $H_{\mathbb{Z}}(a, b)$ in the metric topology of $\ell^{p_{3}}(\mathbb{Z})$.

Proof. In view of Theorem 1.4, it suffices to show that for each $m \in \mathbb{Z}$, the series on the right of (1.27) converges absolutely. This follows immediately by applying Corollary 2.4 to the sequence of $L^{1}(\mathbb{R})$ kernels $\left\{K_{j}\right\}_{j=1}^{\infty}$ specified in (1.25).

Remark 1.3. For a different kind of discrete model for Theorem 1.2 and for the bilinear Hilbert transform for $\mathbb{R}$, see Thiele's results in terms of Walsh wave packets in [30], [31], and [32].

The subsequent sections of this article will be organized as follows. Section 2 is devoted to proving the discretization theorem (Theorem 1.1) and its auxiliary consequence in Corollary 2.4. In order to avoid later digressions, we recall in Section 3 the requisite measure-theoretic background items-focusing on the tools from ergodic operator theory that will be used in Sections 4,5, and 6 for the transference to Lebesgue spaces of bisublinear maximal operators. Theorem 4.1 is a general vehicle for transferring discrete bisublinear maximal operators. Following on this, Sections 4 and 5 include applications of specific transferred discrete bisublinear maximal operators to almost everywhere convergence. Section 4 ends with a brief description of the relevance to the a.e. convergence in Bourgain's double recurrence theorem ([8]), while Section 5 establishes the a.e. convergence of the discrete ergodic bilinear Hilbert averages defined by any translation of an arbitrary locally compact abelian group (Theorem 5.1). The transference of strong type boundedness of discrete maximal bisublinear operators is performed in Sections 4 and 5 via surjective 
isometries of Lebesgue spaces, and in the last section (Section 6) we expand this framework by developing a general approach for transferring bisublinear maximal estimates from an arbitrary locally compact abelian group $\mathcal{G}$ to Lebesgue spaces of sigma-finite measures via representations of $\mathcal{G}$ by separation-preserving bijections of Lebesgue spaces.

In all that follows, we shall employ the following notation. For a given measure $\mu$ and $0<p \leq \infty$, the algebra of all continous linear mappings of $L^{p}(\mu)$ into $L^{p}(\mu)$ will be designated by $\mathfrak{B}\left(L^{p}(\mu)\right)$. The set of non-negative real numbers will be denoted by $\mathbb{R}_{+}$, and the signum function on the complex plane $\mathbb{C}$ will be denoted by $\operatorname{sgn}(\cdot)$. For an arbitrary measure space $(\Omega, \mu)$, the algebra (under pointwise operations) consisting of all complex-valued $\mu$-measurable functions on $\Omega$ (identified modulo equality $\mu$-a.e. on $\Omega$ ) will be symbolized by $\mathcal{A}(\mu)$. Given two complex-valued functions $f$ and $g$ on a set $Y$, we shall, whenever convenient to avoid confusion, denote their pointwise product on $Y$ by $f \cdot g$. If $A$ is a subset of $Y$, then, except where otherwise indicated, the characteristic function of $A$ will be designated by $\chi_{A}$, and the restriction to $A$ of a function $F$ defined on $Y$ will be written $F \mid A$. The symbol $C$ with a (possibly empty) set of subscripts will stand for a constant which depends only on those subscripts, and which can change in value from one occurrence to another.

\section{Proof of Theorem 1.1}

This section is devoted to the demonstration of Theorem 1.1, which will be facilitated by the following lemmas.

Lemma 2.1. Suppose that the exponents $p_{1}, p_{2}$, and $p_{3}$ satisfy (1.6) and (1.7), and let $p^{*}=\min \left(p_{3}, 1\right)$ (hence, in particular $\ell^{p^{*}}(\mathbb{Z}) \subseteq \ell^{1}(\mathbb{Z})$ ). If $\Lambda$ is a sequence of complex numbers belonging to $\ell^{p^{*}}(\mathbb{Z})$, then $\mathfrak{S}_{\Lambda}$ is a bilinear mapping from $\ell^{p_{1}}(\mathbb{Z}) \times$ $\ell^{p_{2}}(\mathbb{Z})$ to $\ell^{p_{3}}(\mathbb{Z})$ which satisfies

$$
\begin{array}{r}
\left\|\mathfrak{S}_{\Lambda}(a, b)\right\|_{\ell^{p_{3}}(\mathbb{Z})} \leq\|\Lambda\|_{\ell^{p^{*}}(\mathbb{Z})}\|a\|_{\ell^{p_{1}}(\mathbb{Z})}\|b\|_{\ell^{p_{2}}(\mathbb{Z})}, \\
\text { for all } a \in \ell^{p_{1}}(\mathbb{Z}), \text { and all } b \in \ell^{p_{2}}(\mathbb{Z}) .
\end{array}
$$

Proof. If either or both of the exponents $p_{1}, p_{2}$ is infinite, then it is easy to see that (2.1 holds. So we now assume that the exponents $p_{1}, p_{2}$, and $p_{3}$ are all finite, and that $a \in \ell^{p_{1}}(\mathbb{Z}), b \in \ell^{p_{2}}(\mathbb{Z})$.. If $p_{3} \geq 1$, then $p^{*}=1$. Moreover, it follows from Minkowski's inequality that

$$
\begin{aligned}
& \left\{\sum_{m=-\infty}^{\infty}\left(\sum_{n=-\infty}^{\infty}\left|a_{m+n}\right|\left|b_{m-n}\right|\left|\Lambda_{n}\right|\right)^{p_{3}}\right\}^{1 / p_{3}} \\
\leq & \sum_{n=-\infty}^{\infty}\left(\sum_{m=-\infty}^{\infty}\left|a_{m+n}\right|^{p_{3}}\left|b_{m-n}\right|^{p_{3}}\left|\Lambda_{n}\right|^{p_{3}}\right)^{1 / p_{3}} \\
= & \sum_{n=-\infty}^{\infty}\left|\Lambda_{n}\right|\left(\sum_{m=-\infty}^{\infty}\left|a_{m+n}\right|^{p_{3}}\left|b_{m-n}\right|{ }^{p_{3}}\right)^{1 / p_{3}} \\
\leq & \left(\sum_{n=-\infty}^{\infty}\left|\Lambda_{n}\right|\right)\|a\|_{\ell^{p_{1}}(\mathbb{Z})}\|b\|_{\ell^{p_{2}}(\mathbb{Z})} .
\end{aligned}
$$


If $p_{3}<1$, then we have

$$
\begin{aligned}
& \sum_{m=-\infty}^{\infty}\left(\sum_{n=-\infty}^{\infty}\left|a_{m+n}\right|\left|b_{m-n}\right|\left|\Lambda_{n}\right|\right)^{p_{3}} \\
\leq & \sum_{m=-\infty}^{\infty} \sum_{n=-\infty}^{\infty}\left|a_{m+n}\right|^{p_{3}}\left|b_{m-n}\right|^{p_{3}}\left|\Lambda_{n}\right|^{p_{3}} \\
= & \sum_{n=-\infty}^{\infty}\left|\Lambda_{n}\right|^{p_{3}} \sum_{m=-\infty}^{\infty}\left|a_{m+n}\right|^{p_{3}}\left|b_{m-n}\right|^{p_{3}} .
\end{aligned}
$$

An application of Hölder's inequality (for the conjugate exponents $p_{1} / p_{3}$ and $p_{2} / p_{3}$ ) to the sum on $m$ in the last expression shows that

$$
\begin{aligned}
& \sum_{m=-\infty}^{\infty}\left(\sum_{n=-\infty}^{\infty}\left|a_{m+n}\right|\left|b_{m-n}\right|\left|\Lambda_{n}\right|\right)^{p_{3}} \\
\leq & \left(\sum_{n=-\infty}^{\infty}\left|\Lambda_{n}\right|^{p_{3}}\right)\|a\|_{\ell^{p_{1}(\mathbb{Z})}}^{p_{3}}\|b\|_{\ell^{p_{2}(\mathbb{Z})}}^{p_{3}},
\end{aligned}
$$

which completes the proof of the lemma, since $p^{*}=p_{3}$ because we are in the case $p_{3}<1$.

It will now be convenient to introduce a few auxiliary notions. For each $\phi \in$ $L^{1}(\mathbb{R})$ such that the support of $\phi$ is a subset of $\mathcal{I}=\left[-\frac{1}{4}, \frac{1}{4}\right]$, we define the linear mapping $P_{\phi}: \mathbb{C}^{\mathbb{Z}} \rightarrow \mathbb{C}^{\mathbb{R}}$ by putting

$$
\begin{aligned}
& \left(P_{\phi}\left(\left\{a_{n}\right\}_{n=-\infty}^{\infty}\right)\right)(x)=\sum_{n \in \mathbb{Z}} a_{n} \phi(x-n), \\
& \quad \text { for all }\left\{a_{n}\right\}_{n=-\infty}^{\infty} \in \mathbb{C}^{\mathbb{Z}}, \text { and all } x \in \mathbb{R} .
\end{aligned}
$$

When $\phi$ is specialized to be $\chi_{\mathcal{I}}$, the characteristic function of $\mathcal{I}$, we shall write $P$ rather than $P_{\phi}$. Clearly, if $0<p \leq \infty$, and if $\phi \in L^{1}(\mathbb{R}) \cap L^{p}(\mathbb{R})$ with support contained in $\mathcal{I}$, then

$$
\begin{gathered}
\left\|P_{\phi}\left(\left\{a_{n}\right\}_{n=-\infty}^{\infty}\right)\right\|_{L^{p}(\mathbb{R})}=\|\phi\|_{L^{p}(\mathbb{R})}\left\|\left\{a_{n}\right\}_{n=-\infty}^{\infty}\right\|_{\ell^{p}(\mathbb{Z})}, \\
\text { for all }\left\{a_{n}\right\}_{n=-\infty}^{\infty} \in \ell^{p}(\mathbb{Z}) .
\end{gathered}
$$

Notice also that if $\phi \in L^{1}(\mathbb{R})$ is a non-negative function with support contained in $\mathcal{I}$, if $N \in \mathbb{N}$, if, for $1 \leq j \leq N, a^{(j)} \equiv\left\{a_{n}^{(j)}\right\}_{n=-\infty}^{\infty}$ is a sequence of real numbers, and if we put

$$
a_{n}^{\#}=\sup _{1 \leq j \leq N} a_{n}^{(j)}, \text { for all } n \in \mathbb{Z},
$$

then pointwise on $\mathbb{R}$ we have

$$
P_{\phi}\left(\left\{a_{n}^{\#}\right\}_{n=-\infty}^{\infty}\right)=\sup _{1 \leq j \leq N} P_{\phi}\left(\left\{a_{n}^{(j)}\right\}_{n=-\infty}^{\infty}\right) .
$$

Definition 2.1. For $K \in L^{1}(\mathbb{R})$ and $n \in \mathbb{Z}$, we define the continuous function $\Phi_{n, K}: \mathbb{R} \rightarrow \mathbb{C}$ by writing for each $x \in \mathbb{R}$,

$$
\Phi_{n, K}(x)=\left(\chi_{\mathcal{I}}(x)\right) \int_{(-1 / 4)+|x|}^{(1 / 4)-|x|} K(n+y) d y .
$$


Lemma 2.2. Suppose that $K \in L^{1}(\mathbb{R}), k \in \mathbb{Z}$, and $a$ and $b$ are finitely supported sequences defined on $\mathbb{Z}$. Then if $x \in \mathcal{I}_{k}$. we have

$$
\left(S_{K}(P a, P b)\right)(x)=\sum_{n=-\infty}^{\infty} a_{k+n} b_{k-n} \Phi_{n, K}(x-k) .
$$

Proof. Clearly

$$
\begin{aligned}
\left(S_{K}(P a, P b)\right)(x) & =\sum_{r, s \in \mathbb{Z}} a_{r} b_{s}\left(S_{K}\left(\chi_{\mathcal{I}_{r}}, \chi_{\mathcal{I}_{s}}\right)\right)(x) \\
& =\sum_{r, s \in \mathbb{Z}} a_{r} b_{s} \int_{(\mathcal{I}+r-x) \cap(\mathcal{I}-s+x)} K(y) d y .
\end{aligned}
$$

Elementary considerations with endpoints show that for $r \in \mathbb{Z}, s \in \mathbb{Z}$, the closed intervals $(\mathcal{I}+r-x)$ and $(\mathcal{I}-s+x)$ will intersect in a set of positive Lebesgue measure if and only if

$$
|r+s-2 x|<\frac{1}{2}
$$

Since $x \in \mathcal{I}_{k},(2.8)$ implies that

$$
|r+s-2 k|<1
$$

whence

$$
r+s=2 k .
$$

Hence we can rewrite the last member of (2.7) by taking $r=k+n, s=k-n$ and letting $n$ run through $\mathbb{Z}$. This gives

$$
\begin{aligned}
& \left(S_{K}(P a, P b)\right)(x) \\
= & \sum_{n=-\infty}^{\infty} a_{k+n} b_{k-n} \int_{(\mathcal{I}+k+n-x) \cap(\mathcal{I}-k+n+x)} K(y) d y \\
= & \sum_{n=-\infty}^{\infty} a_{k+n} b_{k-n} \int_{(\mathcal{I}+k-x) \cap(\mathcal{I}-k+x)} K(y+n) d y .
\end{aligned}
$$

It is clear that if $u \in \mathcal{I}$, then

$$
(u+\mathcal{I}) \bigcap(-u+\mathcal{I})=\left[-\frac{1}{4}+|u|, \frac{1}{4}-|u|\right] .
$$

Taking $u=(x-k)$, we can apply this to (2.9) to get (2.6).

Lemma 2.3. Let $N \in \mathbb{N}$, and suppose that $\left\{K_{j}\right\}_{j=1}^{N} \subseteq L^{1}(\mathbb{R})$ is such that for $1 \leq j \leq N$, and each $n \in \mathbb{Z}$, the restriction $\left(K_{j} \mid \mathcal{I}_{n}\right)$ belongs to $C^{1}\left(\mathcal{I}_{n}\right)$. For each $n \in \mathbb{Z}$, let

$$
\Lambda_{n}=\sup \left\{\left|\left(K_{j} \mid \mathcal{I}_{n}\right)^{\prime}(x)\right|: 1 \leq j \leq N, x \in \mathcal{I}_{n}\right\},
$$

and assume that the sequence $\Lambda \equiv\left\{\Lambda_{n}\right\}_{n=-\infty}^{\infty} \in \ell^{1}(\mathbb{Z})$. Let

$$
\begin{array}{r}
\left(S^{(N)}(f, g)\right)(x)=\sup _{1 \leq j \leq N}\left|\left(S_{K_{j}}(f, g)\right)(x)\right|, \\
\text { for all } f, g \in L^{2}(\mathbb{R}), \text { and all } x \in \mathbb{R},
\end{array}
$$


and put

$$
\begin{gathered}
\left(\mathfrak{S}^{(N)}(\mathfrak{a}, \mathfrak{b})\right)(m)=\sup _{1 \leq j \leq N}\left|\sum_{n=-\infty}^{\infty} \mathfrak{a}_{m+n} \mathfrak{b}_{m-n} K_{j}(n)\right| \\
\text { for all } \mathfrak{a}, \mathfrak{b} \in \ell^{2}(\mathbb{Z}), \text { and all } m \in \mathbb{Z}
\end{gathered}
$$

Further, let $\phi_{0} \geq 0$ and $\phi_{1} \geq 0$ be the functions defined on $\mathbb{R}$ by writing for each $u \in \mathbb{R}$,

$$
\begin{aligned}
& \phi_{0}(u)=2\left(\frac{1}{4}-|u|\right) \chi_{\mathcal{I}}(u) ; \\
& \phi_{1}(u)=\left(\frac{1}{4}-|u|\right)^{2} \chi_{\mathcal{I}}(u) .
\end{aligned}
$$

Then for every pair $a, b$ of finitely supported complex-valued sequences defined on $\mathbb{Z}$, the following inequality holds pointwise on $\mathbb{R}$.

$$
P_{\phi_{0}}\left(\mathfrak{S}^{(N)}(a, b)\right) \leq S^{(N)}(P a, P b)+P_{\phi_{1}}\left(\mathfrak{S}_{\Lambda}(|a|,|b|)\right) .
$$

Proof. First of all we observe that by (2.4) it suffices to establish (2.12) in the special case $N=1$. So without loss of generality we now replace $\left\{K_{j}\right\}_{j=1}^{N}$ by a single kernel $K$, and adjust the notation accordingly. Next observe that for any sequence $w \equiv\left\{w_{n}\right\}_{n=-\infty}^{\infty}$ of complex numbers, $P_{\phi_{0}}(w)$ vanishes on the complement of the union of the intervals $\mathcal{I}_{n}, n \in \mathbb{Z}$. Consequently, we now fix $k \in \mathbb{Z}$, and $x \in \mathcal{I}_{k}$, and we shall complete the proof by showing that (2.12) holds at $x$. For each $n \in \mathbb{Z}$, we have

$$
\begin{aligned}
& \Phi_{n, K}(x-k) \\
= & \phi_{0}(x-k) K(n)+\int_{-\frac{1}{4}+|x-k|}^{\frac{1}{4}-|x-k|}\{K(y+n)-K(n)\} d y .
\end{aligned}
$$

Moreover, as in (1.5), the Fundamental Theorem of Calculus shows that for each $y \in\left[-\frac{1}{4}+|x-k|, \frac{1}{4}-|x-k|\right]$, we have

$$
|K(y+n)-K(n)| \leq A_{n}|y|
$$


Using (2.13) and (2.14), we find that

$$
\begin{aligned}
& \left(P_{\phi_{0}}\left(\mathfrak{S}^{(1)}(a, b)\right)\right)(x) \\
= & \left|\sum_{n=-\infty}^{\infty} a_{k+n} b_{k-n} K(n) \phi_{0}(x-k)\right| \\
\leq & \left|\sum_{n=-\infty}^{\infty} a_{k+n} b_{k-n} \Phi_{n, K}(x-k)\right| \\
& \quad+\left|\sum_{n=-\infty}^{\infty} a_{k+n} b_{k-n}\left(\Phi_{n, K}(x-k)-\phi_{0}(x-k) K(n)\right)\right| \\
\leq & \left|\sum_{n=-\infty}^{\infty} a_{k+n} b_{k-n} \Phi_{n, K}(x-k)\right|+\sum_{n=-\infty}^{\infty}\left|a_{k+n}\right|\left|b_{k-n}\right| \Lambda_{n} \phi_{1}(x-k) \\
= & \left|\sum_{n=-\infty}^{\infty} a_{k+n} b_{k-n} \Phi_{n, K}(x-k)\right|+\left(P_{\phi_{1}}\left(\mathfrak{S}_{\Lambda}(|a|,|b|)\right)\right)(x) .
\end{aligned}
$$

An appeal to Lemma 2.2 now completes the proof of Lemma 2.3 .

Proof of Theorem 1.1. We first observe that conclusion (i) is an immediate consequence of Remark 1.1. In order to obtain conclusion (ii) temporarily fix an arbitrary $N \in \mathbb{N}$, and let $a \equiv\left\{a_{n}\right\}_{n=-\infty}^{\infty}, b \equiv\left\{b_{n}\right\}_{n=-\infty}^{\infty}$ be finitely supported sequences of complex numbers. By the hypothesis in (1.8), followed by use of (2.3), we have, in the notation of Lemma 2.3,

$$
\begin{aligned}
\left\|S^{(N)}(P a, P b)\right\|_{L^{p_{3}(\mathbb{R})}} & \leq \mathfrak{C}\|P a\|_{L^{p_{1}(\mathbb{R})}}\|P b\|_{L^{p_{2}(\mathbb{R})}} \\
& =\mathfrak{C} 2^{-1 / p_{3}}\|a\|_{\ell^{p_{1}}(\mathbb{Z})}\|b\|_{\ell^{p_{2}(\mathbb{Z})}} .
\end{aligned}
$$

It is evident from the definition in $(2.11)$ that $\left\|\phi_{1}\right\|_{L^{p_{3}(\mathbb{R})}} \leq \frac{1}{16}$, and so from Lemma 2.1 and (2.3) we see that

$$
\left\|P_{\phi_{1}}\left(\mathfrak{S}_{\Lambda}(|a|,|b|)\right)\right\|_{L^{p_{3}(\mathbb{R})}} \leq \frac{\|A\|_{\ell^{p^{*}}(\mathbb{Z})}\|a\|_{\ell^{p_{1}}(\mathbb{Z})}\|b\|_{\ell^{p_{2}(\mathbb{Z})}}}{16} .
$$

From (2.12) we infer that $\left\|P_{\phi_{0}}\left(\mathfrak{S}^{(N)}(a, b)\right)\right\|_{L^{p_{3}(\mathbb{R})}}=\left\|\phi_{0}\right\|_{L^{p_{3}(\mathbb{R})}}\left\|\mathfrak{S}^{(N)}(a, b)\right\|_{\ell^{p_{3}}(\mathbb{Z})}$ does not exceed

$$
\begin{array}{cc}
\left\|S^{(N)}(P a, P b)\right\|_{L^{p_{3}(\mathbb{R})}}+\left\|P_{\phi_{1}}\left(\mathfrak{S}_{\Lambda}(|a|,|b|)\right)\right\|_{L^{p_{3}(\mathbb{R})}}, & \text { if } 1 \leq p_{3} \leq \infty ; \\
\left(\left\|S^{(N)}(P a, P b)\right\|_{L^{p_{3}(\mathbb{R})}}^{p^{2}}+\left\|P_{\phi_{1}}\left(\mathfrak{S}_{\Lambda}(|a|,|b|)\right)\right\|_{L^{p_{3}(\mathbb{R})}}^{p_{3}}\right)^{1 / p_{3}}, & \text { if } 0<p_{3}<1 .
\end{array}
$$

In view of (2.15) and (2.16) these two estimates show that there is a constant $\eta$ depending only on $p_{1}, p_{2}$, and $\left\{K_{j}\right\}_{j=1}^{\infty}$. such that for arbitrary $N \in \mathbb{N}$ and for arbitrary finitely supported sequences $a \equiv\left\{a_{n}\right\}_{n=-\infty}^{\infty}, b \equiv\left\{b_{n}\right\}_{n=-\infty}^{\infty}$,

$$
\left\|\mathfrak{S}^{(N)}(a, b)\right\|_{\ell^{p_{3}(\mathbb{Z})}} \leq \eta\|a\|_{\ell^{p_{1}}(\mathbb{Z})}\|b\|_{\ell^{p_{2}(\mathbb{Z})}} .
$$

Now let $a \equiv\left\{a_{n}\right\}_{n=-\infty}^{\infty}, b \equiv\left\{b_{n}\right\}_{n=-\infty}^{\infty}$ be arbitrary vectors in $\ell^{p_{1}}(\mathbb{Z})$ and $\ell^{p_{2}}(\mathbb{Z})$, respectively. For arbitrary $L \in \mathbb{N}$, denote by $\xi_{L}$ the characteristic function, defined 
on $\mathbb{Z}$, of $\{k \in \mathbb{Z}:|k| \leq L\}$, and define the finitely supported sequences $a^{(L)}$ and $b^{(L)}$ by writing for each $n \in \mathbb{Z}$,

$$
\begin{aligned}
& a_{n}^{(L)}=a_{n} \xi_{L}(n) \\
& b_{n}^{(L)}=b_{n} \xi_{L}(n) .
\end{aligned}
$$

Observe that for $N \in \mathbb{N}$, and each $m \in \mathbb{Z}$, we have, for $1 \leq j \leq N$,

$$
\sum_{n=-\infty}^{\infty} a_{m+n}^{(L)} b_{m-n}^{(L)} K_{j}(n)=\sum_{n=\max (-m-L, m-L)}^{\min (-m+L, m+L)} a_{m+n} b_{m-n} K_{j}(n),
$$

and it follows that as $L \rightarrow \infty$,

$$
\mathfrak{S}^{(N)}\left(a^{(L)}, b^{(L)}\right) \rightarrow \mathfrak{S}^{(N)}(a, b) \text { pointwise on } \mathbb{Z}
$$

Consequently, (2.17) remains valid for all $a \equiv\left\{a_{n}\right\}_{n=-\infty}^{\infty} \in \ell^{p_{1}}(\mathbb{Z})$, and all $b$ $\equiv\left\{b_{n}\right\}_{n=-\infty}^{\infty} \in \ell^{p_{2}}\left(\mathbb{Z}\right.$ ) (if $p_{3}<\infty$, use Fatou's Lemma together with (2.17) applied to $\mathfrak{S}^{(N)}\left(a^{(L)}, b^{(L)}\right)$, and if $p_{1}=p_{2}=p_{3}=\infty$, this estimate follows directly from (2.17) together with (2.18)). Thereafter, we can let $N \rightarrow \infty$ in the resulting version of (2.17) in order to obtain (1.11) in similar fashion (using the monotone convergence theorem when $\left.p_{3}<\infty\right)$.

We close this section with the following immediate corollary of Theorem 1.1.

Corollary 2.4. Under the hypotheses of Theorem 1.1, the constant $\eta$ in (1.11) has the following property: for all $a \equiv\left\{a_{n}\right\}_{n=-\infty}^{\infty} \in \ell^{p_{1}}(\mathbb{Z})$, all $b \equiv\left\{b_{n}\right\}_{n=-\infty}^{\infty} \in$ $\ell^{p_{2}}(\mathbb{Z})$, each $j \in \mathbb{N}$, and each $m \in \mathbb{Z}$,

$$
\sum_{n=-\infty}^{\infty}\left|a_{m+n}\right|\left|b_{m-n}\right|\left|K_{j}(n)\right| \leq \eta\|a\|_{\ell^{p_{1}(\mathbb{Z})}}\|b\|_{\ell^{p_{2}(\mathbb{Z})}} .
$$

Proof. Define the sequences $a^{*} \equiv\left\{a_{n}^{*}\right\}_{n=-\infty}^{\infty} \in \ell^{p_{1}}(\mathbb{Z})$, and $b^{*} \equiv\left\{b_{n}^{*}\right\}_{n=-\infty}^{\infty} \in$ $\ell^{p_{2}}(\mathbb{Z})$ by writing for each $n \in \mathbb{Z}$,

$$
\begin{aligned}
a_{n}^{*} & =\left|a_{n}\right|\left(\operatorname{sgn}\left(K_{j}(n-m)\right)\right) \\
b_{n}^{*} & =\left|b_{n}\right| .
\end{aligned}
$$

Using (1.11) we see that

$$
\begin{aligned}
\sum_{n=-\infty}^{\infty}\left|a_{m+n}\right|\left|b_{m-n}\right|\left|K_{j}(n)\right| & =\sum_{n=-\infty}^{\infty} a_{m+n}^{*} b_{m-n}^{*} K_{j}(n) \\
& \leq\left\|\mathfrak{S}_{\{\mathcal{K}(j)\}}^{b}\left(a^{*}, b^{*}\right)\right\|_{\ell^{p_{3}(\mathbb{Z})}} \leq \eta\|a\|_{\ell^{p_{1}(\mathbb{Z})}}\|b\|_{\ell^{p_{2}(\mathbb{Z})}} .
\end{aligned}
$$




\section{Measure-Theoretic Background Items}

In this section we collect the necessary tools for the transference to measure spaces of bisublinear maximal estimates and almost everywhere convergence of bilinear mappings. For the almost everywhere convergence aspects, we shall require the following multilinear version of the Banach Principle (a calculational proof of this multilinear Banach Principle is indicated in the reasoning for Theorem 6 of [18]; aternatively, its demonstration can be carried out directly by induction on $m$, since for $m=1$ the proposition asserts the standard Banach Principle for linear operators).

Proposition 3.1. Let $m \in \mathbb{N}$, and let $(\Gamma, \sigma)$ and $\left(\Omega_{j}, \mu_{j}\right), 1 \leq j \leq m$, be arbitrary measure spaces. Suppose that for $1 \leq j \leq m, p_{j}$ is a positive real number, and $\mathfrak{S}_{j}$ is a dense subset of $L^{p_{j}}\left(\mu_{j}\right)$. Assume that $\left\{T_{k}\right\}_{k=1}^{\infty}$ is a sequence of multilinear mappings of $\prod_{j=1}^{m} L^{p_{j}}\left(\mu_{j}\right)$ into the algebra $\mathcal{A}(\sigma)$, consisting of all complex-valued $\sigma$-measurable functions on $\Gamma$ (identified modulo equality $\sigma$-a.e. on $\Gamma$ ), and for all $\left(f_{1}, f_{2}, \cdots, f_{m}\right) \in \prod_{j=1}^{m} L^{p_{j}}\left(\mu_{j}\right)$ and all $x \in \Gamma$, put

$$
\left(T_{*}\left(f_{1}, f_{2}, \cdots, f_{m}\right)\right)(x)=\sup _{k \in \mathbb{N}}\left|\left(T_{k}\left(f_{1}, f_{2}, \cdots, f_{m}\right)\right)(x)\right| .
$$

Suppose further that there are a constant $B$ and a positive real number $r$ such that

$$
\begin{aligned}
& \sigma\left\{x \in \Gamma:\left(T_{*}\left(f_{1}, f_{2}, \cdots, f_{m}\right)\right)(x)>y\right\} \leq\left(\left(\frac{B}{y}\right) \prod_{j=1}^{m}\left\|f_{j}\right\|_{L^{p_{j}}\left(\mu_{j}\right)}\right)^{r}, \\
& \quad \text { for all positive real numbers } y \text {, and all }\left(f_{1}, f_{2}, \cdots, f_{m}\right) \in \prod_{j=1}^{m} L^{p_{j}}\left(\mu_{j}\right) .
\end{aligned}
$$

If for every $\left(g_{1}, g_{2}, \cdots, g_{m}\right) \in \prod_{j=1}^{m} \mathfrak{S}_{j}$,

$$
\lim _{k}\left(T_{k}\left(g_{1}, g_{2}, \cdots, g_{m}\right)\right)(x)
$$

exists in $\mathbb{C}$ for $\sigma$-almost all $x \in \Gamma$, then likewise for all $\left(f_{1}, f_{2}, \cdots, f_{m}\right) \in \prod_{j=1}^{m} L^{p_{j}}\left(\mu_{j}\right)$,

$$
\lim _{k}\left(T_{k}\left(f_{1}, f_{2}, \cdots, f_{m}\right)\right)(x)
$$

exists in $\mathbb{C}$ for $\sigma$-almost all $x \in \Gamma$.

For the transference to measure spaces of strong type maximal estimates defined by families of multilinear operators, we now recall key facts regarding the structure of separation-preserving linear operators. (For the transference of strong type maximal estimates defined by sequences of linear operators, see, e.g., [1] and Theorem (2.11) of [6].)

Definition 3.1. Let $(\Omega, \mu)$ be an arbitrary measure space, and suppose that $0<p<$ $\infty$. A continuous linear mapping $\mathcal{S}$ of $L^{p}(\mu)$ into $L^{p}(\mu)$ will be called separationpreserving provided that whenever $f \in L^{p}(\mu), g \in L^{p}(\mu)$ and the pointwise product $f g$ vanishes $\mu$-a.e. on $\Omega$, it follows that the pointwise product $(\mathcal{S} f)(\mathcal{S} g)$ vanishes $\mu$-a.e. on $\Omega$. A continuous linear mapping $\mathfrak{P}$ of $L^{p}(\mu)$ into $L^{p}(\mu)$ will be called positive provided that $\mathfrak{P} f \geq 0 \mu$-a.e. on $\Omega$ whenever $f \in L^{p}(\mu)$ and $f \geq 0 \mu$-a.e. on $\Omega$.

In the literature separation-preserving operators are also called Lamperti or disjointness-preserving (see [24] for a full account of their basic features). The 
proof of Theorem 3.1 in [24] is valid for $(\Omega, \mu)$ and $p$ as in Definition 3.1, and furnishes the following characterization of the separation-preserving property.

Theorem 3.2. Suppose that $(\Omega, \mu)$ is an arbitrary measure space, $0<p<\infty$, and $T$ is a continuous linear mapping of $L^{p}(\mu)$ into $L^{p}(\mu)$. Then $T$ is separationpreserving if and only if there is a continuous linear transformation $|T|$ mapping $L^{p}(\mu)$ into $L^{p}(\mu)$ such that $|T|$ is positive, and

$$
|T f|=|T|(|f|), \text { for all } f \in L^{p}(\mu) .
$$

If this is the case, then (3.1) characterizes $|T|$ among the continuous linear mappings of $L^{p}(\mu)$ into $L^{p}(\mu)$ (we then call $|T|$ the "linear modulus" of $T$ ). Moreover, $|T|$ is separation-preserving, and satisfies

$$
|T f|=|| T|(f)|, \text { for all } f \in L^{p}(\mu) .
$$

(Hence for all $f \in L^{p}(\mu),\|T f\|_{L^{p}(\mu)}=\||T|(|f|)\|_{L^{p}(\mu)}=\||T|(f)\|_{L^{p}(\mu)}$.)

Application of (3.1) to the functions $f \geq 0$ in $L^{p}(\mu)$ yields the following obvious corollary.

Corollary 3.3. If $(\Omega, \mu)$ is an arbitrary measure space, $0<p<\infty$, and $T$ is a continuous linear mapping of $L^{p}(\mu)$ into $L^{p}(\mu)$ such that $T$ is separation-preserving, then $T$ is positive if and only if $T=|T|$.

Since $|T|$ is a positive operator on $L^{p}(\mu),|T|$ maps the class of real-valued functions belonging to $L^{p}(\mu)$ into itself. The following basic corollary of Theorem 3.2 already indicates the utility of separation-preserving operators for the transference of maximal estimates.

Corollary 3.4. Suppose that $(\Omega, \mu)$ is an arbitrary measure space, $0<p<\infty$, and $T$ is a continuous linear mapping of $L^{p}(\mu)$ into $L^{p}(\mu)$ such that $T$ is separationpreserving. Suppose further that $N \in \mathbb{N}$, and that $\left\{f_{j}\right\}_{j=1}^{N} \subseteq L^{p}(\mu)$, with $f_{j}$ realvalued for $1 \leq j \leq N$. Then $|T|$, the linear modulus of $T$, satisfies:

$$
\begin{aligned}
& |T|\left(\sup _{1 \leq j \leq N} f_{j}\right)=\sup _{1 \leq j \leq N}\left(|T|\left(f_{j}\right)\right) ; \\
& |T|\left(\inf _{1 \leq j \leq N} f_{j}\right)=\inf _{1 \leq j \leq N}\left(|T|\left(f_{j}\right)\right) .
\end{aligned}
$$

Proof. The case $N=1$ is trivial. In the case $N=2$, we have:

$$
\begin{aligned}
\sup _{1 \leq j \leq 2} f_{j} & =\frac{\left|f_{1}-f_{2}\right|+f_{1}+f_{2}}{2} ; \\
\inf _{1 \leq j \leq 2} f_{j} & =\frac{f_{1}+f_{2}-\left|f_{1}-f_{2}\right|}{2} .
\end{aligned}
$$

Applying $|T|$ to (3.5) and (3.6), we see with the aid of the general properties of $|T|$ asserted by (3.1) and (3.2) that

$$
\begin{aligned}
& |T|\left(\sup _{1 \leq j \leq 2} f_{j}\right)=\frac{|| T\left|f_{1}-\right| T\left|f_{2}\right|+|T| f_{1}+|T| f_{2}}{2}=\sup _{1 \leq j \leq 2}\left(|T| f_{j}\right) \\
& |T|\left(\inf _{1 \leq j \leq 2} f_{j}\right)=\frac{|T| f_{1}+|T| f_{2}-|| T\left|f_{1}-\right| T\left|f_{2}\right|}{2}=\inf _{1 \leq j \leq 2}\left(|T| f_{j}\right) .
\end{aligned}
$$

The desired conclusions in (3.3) and (3.4) follow by induction on $N$ by utilizing the case for $N=2$ in (3.7) and (3.8). 
Recall that by the closed graph theorem for F-spaces (see, eg., Theorem II.2.2 of [16]) if $0<p<\infty, \mu$ is an arbitrary measure, and $Q$ is a one-to-one continuous linear mapping of $L^{p}(\mu)$ onto $L^{p}(\mu)$, then $Q^{-1}$ is also a continuous linear mapping of $L^{p}(\mu)$ onto $L^{p}(\mu)$. This fact is taken for granted in the following well-known proposition relating the separation-preserving property and invertibility (for a proof of this proposition, see, e.g., the demonstration of Scholium (2.3) in [3], where the reasoning is valid for $0<p<\infty)$.

Proposition 3.5. Suppose that $(\Omega, \mu)$ is an arbitrary measure space, $0<p<\infty$, and $T$ is a one-to-one continuous linear mapping of $L^{p}(\mu)$ onto $L^{p}(\mu)$ such that $T$ is separation-preserving. Then $T^{-1}$ is separation-preserving, $|T|$ is a one-to-one continuous linear mapping of $L^{p}(\mu)$ onto $L^{p}(\mu)$, and

$$
|T|^{-1}=\left|T^{-1}\right| \text {. }
$$

Remark 3.1. It follows readily from (3.1) and (3.9) that if the hypotheses of Proposition 3.5 hold, then for all $n \in \mathbb{Z}, T^{n}$ is separation-preserving, and

$$
\left|T^{n}\right|=|T|^{n} .
$$

We next describe two important classes of separation-preserving operators in the setting of an arbitrary measure $\mu$, and $0<p<\infty$. If $\mathcal{S}$ is a continuous, positive, invertible linear mapping of $L^{p}(\mu)$ onto $L^{p}(\mu)$, then $\mathcal{S}$ is separation-preserving if and only if $\mathcal{S}^{-1}$ is positive (the "only if" part follows from (3.9), while the proof of Proposition 3.1 in [24] covers the "if" part in the generality stated here). The second special class of separation-preserving operators is specified by the following proposition, which follows directly from Corollary 2.1 of [29].

Proposition 3.6. Suppose that $(\Omega, \mu)$ is an arbitrary measure space, $0<p<\infty$, and $p \neq 2$. If $U$ is a linear isometry of $L^{p}(\mu)$ into $L^{p}(\mu)$, then $U$ is separationpreserving.

As pointed out by C-H Kan ([24]), when we confine our attention to sigma-finite measure spaces, the following major structural theorem for separation-preserving operators results (see the reasoning used for Theorem 4.1 in [24], which relies on adjustments to both the proof of Theorem 3.1 in [29] and the discussion on pages 453,454 of [15]-this approach furnishes the range $0<p<\infty$ stated here).

Proposition 3.7. Suppose that $(\Omega, \mu)$ is a sigma-finite measure space, and $0<$ $p<\infty$. Denote by $\mathcal{A}(\mu)$ the algebra under pointwise operations consisting of all complex-valued $\mu$-measurable functions on $\Omega$ (identified modulo equality $\mu$-a.e. on $\Omega)$, and let $T$ be a linear, continuous, separation-preserving bijection of $L^{p}(\mu)$ onto $L^{p}(\mu)$. Then there are sequences $\left\{h_{j}\right\}_{j=-\infty}^{\infty}$ and $\left\{\Phi_{j}\right\}_{j=-\infty}^{\infty}$ such that for each $j \in \mathbb{Z}$ : (i) $h_{j} \in \mathcal{A}(\mu)$, with $\left|h_{j}\right|>0$ on $\Omega$, and $\Phi_{j}$ is an algebra automorphism of $\mathcal{A}(\mu)$ onto $\mathcal{A}(\mu)$; (ii) for every $f \in L^{p}(\mu), T^{j} f$ is expressed by the pointwise product on $\Omega$ of the functions $h_{j}$ and $\Phi_{j}(f)$; (iii) whenever $\left\{f_{k}\right\}_{k=1}^{\infty} \subseteq \mathcal{A}(\mu), f \in \mathcal{A}(\mu)$, and $f_{k} \rightarrow f \mu$-a.e. on $\Omega$, it follows that as $k \rightarrow \infty, \Phi_{j}\left(f_{k}\right) \rightarrow \Phi_{j}(f) \mu$-a.e. on $\Omega$. The sequences $\left\{h_{j}\right\}_{j=-\infty}^{\infty}$ and $\left\{\Phi_{j}\right\}_{j=-\infty}^{\infty}$ are uniquely determined by these properties.

The sequences $\left\{h_{j}\right\}_{j=-\infty}^{\infty}$ and $\left\{\Phi_{j}\right\}_{j=-\infty}^{\infty}$ in Proposition 3.7 are said to be associated with $T$. It is easily seen that for each $j \in \mathbb{Z}, \Phi_{j}(g) \geq 0$ for each $g \geq 0$ belonging to $\mathcal{A}(\mu)$, and that for $f \in \mathcal{A}(\mu)$, and $0<\alpha<\infty$, we have

$$
\left|\Phi_{j}(f)\right|^{\alpha}=\Phi_{j}\left(|f|^{\alpha}\right) \text {. }
$$


Application of the group property $T^{j+k}=T^{j} T^{k}$ to the uniquely determined sequences $\left\{h_{j}\right\}_{j=-\infty}^{\infty}$ and $\left\{\Phi_{j}\right\}_{j=-\infty}^{\infty}$ associated with $T$ furnishes the following relationships, valid for all $j \in \mathbb{Z}, k \in \mathbb{Z}$.

$$
\begin{aligned}
\Phi_{j+k}(f) & =\Phi_{j}\left(\Phi_{k}(f)\right), \text { for every } f \in \mathcal{A}(\mu) . \\
h_{j+k}(x) & =h_{j}(x)\left(\Phi_{j} h_{k}\right)(x), \text { for } \mu \text {-almost all } x \in \Omega .
\end{aligned}
$$

The following version of Corollary 3.1 in [29] will be useful when we use Lebesgue space isometries to transfer the boundedness results of $\S \S 1,2$ for discrete bisublinear maximal operators.

Proposition 3.8. Suppose that $(\Omega, \mu)$ is a sigma-finite measure space, and $0<$ $\alpha, \beta \leq \infty$, with $\alpha \neq \beta$. Let $U$ be a bijective linear mapping of $\mathcal{A}(\mu)$ onto $\mathcal{A}(\mu)$ such that the following two conditions hold.

(i) Whenever $\left\{g_{k}\right\}_{k=1}^{\infty} \subseteq \mathcal{A}(\mu), g \in \mathcal{A}(\mu)$, and $g_{k} \rightarrow g \mu$-a.e. on $\Omega$, it follows that as $k \rightarrow \infty, U\left(g_{k}\right) \rightarrow U(g) \mu$-a.e. on $\Omega$, and $U^{-1}\left(g_{k}\right) \rightarrow U^{-1}(g)$ $\mu$-a.e. on $\Omega$.

(ii) The restrictions $\left(U \mid L^{\alpha}(\mu)\right)$ and $\left(U \mid L^{\beta}(\mu)\right)$ are surjective linear isometries of $L^{\alpha}(\mu)$ and $L^{\beta}(\mu)$, respectively.

Then there are unique sequences $\left\{h_{j}\right\}_{j=-\infty}^{\infty}$ and $\left\{\Phi_{j}\right\}_{j=-\infty}^{\infty}$ such that for each $j \in \mathbb{Z}:$

(j) $h_{j} \in \mathcal{A}(\mu)$, with $\left|h_{j}\right|=1$ on $\Omega$, and $\Phi_{j}$ is an algebra automorphism of $\mathcal{A}(\mu)$ onto $\mathcal{A}(\mu)$

(jj) for every $f \in \mathcal{A}(\mu), U^{j} f$ is expressed by the pointwise product on $\Omega$ of the functions $h_{j}$ and $\Phi_{j}(f)$;

(jjj) whenever $\left\{f_{k}\right\}_{k=1}^{\infty} \subseteq \mathcal{A}(\mu), f \in \mathcal{A}(\mu)$, and $f_{k} \rightarrow f \mu$-a.e. on $\Omega$, it follows that as $k \rightarrow \infty, \Phi_{j}\left(f_{k}\right) \rightarrow \Phi_{j}(f) \mu$-a.e. on $\Omega$.

This unique sequence $\left\{\Phi_{j}\right\}_{j=-\infty}^{\infty}$ has the property that

$$
\begin{aligned}
& \mu(E)=\int_{\Omega} \Phi_{j}\left(\chi_{E}\right) d \mu, \\
& \quad \text { for each } j \in \mathbb{Z}, \text { and each } \mu \text {-measurable set } E .
\end{aligned}
$$

In consequence of the foregoing, the restriction $\left(U \mid L^{p}(\mu)\right)$ is a surjective linear isometry of $L^{p}(\mu)$, for $0<p \leq \infty$.

Proof. The contents of this proposition are well-known, and so we shall merely outline its proof. The existence assertions when both $\alpha$ and $\beta$ are finite can be seen from the discussion of Corollary 3.1 in [29]. So we take up the existence asserions for the case where one of $\alpha, \beta$ equals $\infty$. For sake of definiteness, suppose that $\beta=\infty$ and $0<\alpha<\infty$. Since the Gelfand representation of the commutative $C^{*}$-algebra $L^{\infty}(\mu)$ identifies $L^{\infty}(\mu)$ with the Banach algebra $C(\Delta)$ of all continuous complexvalued functions on a compact Hausdorff space $\Delta$ (see, e.g., Theorem (C.28) in [21]), we can apply the Banach-Stone theorem's characterization for the surjective linear isometries of $C(\Delta)$ (see, e.g., Theorem VI.2.1 of [12]) to infer that for each $j \in \mathbb{Z}$

$$
\left(U^{j} \mid L^{\infty}(\mu)\right)(f)=H_{j} \cdot \Psi_{j}(f) \text {, for all } f \in L^{\infty}(\mu),
$$

where $H_{j} \in \mathcal{A}(\mu)$, with $\left|H_{j}\right|=1$ on $\Omega$, and $\Psi_{j}$ is a norm-preserving algebra automorphism of $L^{\infty}(\mu)$ onto $L^{\infty}(\mu)$. Using this, we can invoke the continuity of $U \mid L^{\alpha}(\mu)$ (or, alternatively the hypothesis in (i)) to infer that $U \mid L^{\alpha}(\mu)$ is separation- preserving (even if $\alpha=2$ ). We can thus associate with $U \mid L^{\alpha}(\mu)$ 
the sequences $\left\{h_{j}\right\}_{j=-\infty}^{\infty}$ and $\left\{\Phi_{j}\right\}_{j=-\infty}^{\infty}$ furnished by Proposition 3.7. If $j \in \mathbb{Z}$, and $\mu(E)<\infty$, then $\Phi_{j}\left(\chi_{E}\right)$ and $\Psi_{j}\left(\chi_{E}\right)$ are characteristic functions which the equality

$$
h_{j} \Phi_{j}\left(\chi_{E}\right)=H_{j} \Psi_{j}\left(\chi_{E}\right)
$$

shows must have the same set of zeroes. Hence $\Phi_{j}\left(\chi_{E}\right)=\Psi_{j}\left(\chi_{E}\right)$. By taking absolute values on both sides of (3.15) we can now infer that for $j \in \mathbb{Z}$, and $\mu(E)<\infty$,

$$
\left|h_{j}\right| \Phi_{j}\left(\chi_{E}\right)=\Phi_{j}\left(\chi_{E}\right) .
$$

Since $\mu$ is sigma-finite, and $\Phi_{j}$ preserves $\mu$-a.e. convergence on $\Omega$, this implies that for each $j \in \mathbb{Z},\left|h_{j}\right|=\Phi_{j}(1)=1$ on $\Omega$. The sigma-finiteness of $\mu$ implies that each $f \in \mathcal{A}(\mu)$ is the $\mu$-a.e. limit on $\Omega$ of a sequence of $\mu$-integrable simple functions, and so the desired conclusion in (jj) follows from the fact that

$$
\left(U^{j} \mid L^{\alpha}(\mu)\right)(f)=h_{j} \cdot \Phi_{j}(f), \text { for all } f \in L^{\alpha}(\mu),
$$

since $\Phi_{j}$ preserves $\mu$-a.e. convergence on $\Omega$, and by our hypothesis in (i) so does $U^{j}$. The desired conclusion in (3.14) is readily seen when $\mu(E)<\infty$, since $\left(U^{j} \mid L^{\alpha}(\mu)\right)$ is an isometry of $L^{\alpha}(\mu)$, and $\left|h_{j}\right|=1$ on $\Omega$. The sigma-finiteness of $\mu$ can then be used to remove the requirement that the measure of $E$ is finite, and complete the proof of the existence assertions.

Turning now to the proof of the remaining conclusions for the general case of $\alpha$ and $\beta$, and putting $\delta=\min \{\alpha, \beta\}$, we obtain the uniqueness assertion of the present proposition immediately by applying to $\left(U^{j} \mid L^{\delta}(\mu)\right)$ the uniqueness conclusion in Theorem 3.7. For $0<p<\infty$, it is clear from conclusion $(\mathrm{j})$ and (3.14) that for every $\mu$-integrable simple function $f,\|U f\|_{L^{p}(\mu)}=\left\|U^{-1} f\right\|_{L^{p}(\mu)}=\|f\|_{L^{p}(\mu)}$, and so for $0<p<\infty$, the last conclusion of the present proposition follows by the density of the $\mu$-integrable simple functions in $L^{p}(\mu)$, used in conjunction with hypothesis (i). To show that $\left(U \mid L^{\infty}(\mu)\right)$ is a surjective isometry of $L^{\infty}(\mu)$ it suffices to observe that for $j \in \mathbb{Z}$, and $F \in L^{\infty}(\mu)$, we can infer with the aid of (j), (3.11), and the positivity of $\Phi_{j}$ on $\mathcal{A}(\mu)$ that

$$
\left|U^{j} F\right|=\left|\Phi_{j}(F)\right|=\Phi_{j}(|F|) \leq\|F\|_{L^{\infty}(\mu)},
$$

and so $\left\|U^{j} F\right\|_{L^{\infty}(\mu)} \leq\|F\|_{L^{\infty}(\mu)}$.

Remark 3.2. (i) As has already been indicated above, the unique sequences $\left\{h_{j}\right\}_{j=-\infty}^{\infty}$ and $\left\{\Phi_{j}\right\}_{j=-\infty}^{\infty}$ furnished by the conclusion of Proposition 3.8 clearly coincide with the unique pair of sequences associated by Proposition 3.7 with $\left(U \mid L^{p}(\mu)\right)$ whenever $0<p<\infty$, and so, without ambiguity, we shall expand our terminology by referring to these sequences $\left\{h_{j}\right\}_{j=-\infty}^{\infty}$ and $\left\{\Phi_{j}\right\}_{j=-\infty}^{\infty}$ as being associated with the bijective linear mapping $U$ of $\mathcal{A}(\mu)$ onto $\mathcal{A}(\mu)$ in the hypotheses of Proposition 3.8. In particular, the sequences $\left\{h_{j}\right\}_{j=-\infty}^{\infty}$ and $\left\{\Phi_{j}\right\}_{j=-\infty}^{\infty}$ associated with $U$ satisfy (3.11) through (3.13). (ii) Examples of a bijective linear mapping $U$ of $\mathcal{A}(\mu)$ onto $\mathcal{A}(\mu)$ associated by Proposition 3.8 with a sequence $\left\{h_{j}\right\}_{j=-\infty}^{\infty}$ of non-constant functions can be formed by starting with an invertible measure-preserving mapping $\psi$ of $(\Omega, \mu)$ and a measurable function $u: \Omega \rightarrow \mathbb{T}$, and then setting

$$
(U f)(x) \equiv \frac{u(\psi(x))}{u(x)} f(\psi(x)), \text { for all } f \in \mathcal{A}(\mu) .
$$


When $\mu$ is taken to be Haar measure on a compact abelian group having archimedean ordered dual, then examples of $U$ associated with non-trivial $\left\{h_{j}\right\}_{j=-\infty}^{\infty}$ can be fashioned from the "cocycles" arising in Helson's theory of generalized analyticity and invariant subspaces (for this circle of ideas, see, e.g., [4], [19], and [20]).

The following corollary will be convenient for applying Proposition 3.8 to the transference of maximal operators affiliated with sequences of bilinear operators.

Corollary 3.9. Assume the hypotheses of Proposition 3.8, and let $\left\{h_{j}\right\}_{j=-\infty}^{\infty}$ and $\left\{\Phi_{j}\right\}_{j=-\infty}^{\infty}$ be the sequences thereby associated with $U$. Define the linear bijection $V$ of $\mathcal{A}(\mu)$ onto $\mathcal{A}(\mu)$ by writing

$$
V(f)=h_{1} U(f)=h_{1}^{2} \Phi_{1}(f), \text { for all } f \in \mathcal{A}(\mu) .
$$

Then $V$ also satisfies the hypotheses of Proposition 3.8, which associates with $V$ the sequences $\left\{h_{j}^{2}\right\}_{j=-\infty}^{\infty}$ and $\left\{\Phi_{j}\right\}_{j=-\infty}^{\infty}$. For all $j \in \mathbb{Z}, m \in \mathbb{Z}, n \in \mathbb{Z}, f \in \mathcal{A}(\mu)$, $g \in \mathcal{A}(\mu)$, we have, pointwise on $\Omega$,

$$
V^{j}\left(\left(U^{m} f\right) \cdot\left(U^{n} g\right)\right)=\left(U^{j+m} f\right) \cdot\left(U^{j+n} g\right) .
$$

Proof. Since $\left|h_{1}\right|=1$ on $\Omega$, it is clear that multiplication by $h_{1}$ on $\mathcal{A}(\mu)$ (respectively on $L^{\alpha}(\mu), L^{\beta}(\mu)$ ) is a linear bijection of $\mathcal{A}(\mu)$ (respectively, a surjective linear isometry). Hence $V=h_{1} U$ has these mapping properties as well. Moreover, for all $f \in \mathcal{A}(\mu)$

$$
V^{-1} f=U^{-1}\left(\frac{f}{h_{1}}\right)
$$

These observations show that $V$ satisfies the hypotheses of Proposition 3.8. With the aid of (3.12) (applied to $U$ and to $V$ ), together with the left and right sides of (3.16), it is readily verified that $\left\{\Phi_{j}\right\}_{j=-\infty}^{\infty}$ is also the sequence of $\mathcal{A}(\mu)$-automorphisms associated with $V$. Similar considerations that apply (3.13) to $U$ and to $V$ now show that $\left\{h_{j}^{2}\right\}_{j=-\infty}^{\infty}$ is the sequence of unimodular functions associated with $V$. For all $j \in \mathbb{Z}, m \in \mathbb{Z}, n \in \mathbb{Z}, f \in \mathcal{A}(\mu), g \in \mathcal{A}(\mu)$, we have, pointwise on $\Omega$,

$$
\begin{aligned}
V^{j}\left(\left(U^{m} f\right) \cdot\left(U^{n} g\right)\right) & =h_{j}^{2} \cdot \Phi_{j}\left(U^{m} f\right) \cdot \Phi_{j}\left(U^{n} g\right) \\
& =h_{j}^{2} \cdot \Phi_{j}\left(h_{m} \Phi_{m} f\right) \cdot \Phi_{j}\left(h_{n} \Phi_{n} g\right) \\
& =\left(h_{j} \Phi_{j}\left(h_{m}\right) \Phi_{j+m} f\right)\left(h_{j} \Phi_{j}\left(h_{n}\right) \Phi_{j+n} g\right) .
\end{aligned}
$$

Using (3.13) on the right establishes (3.17) and thereby completes the proof of Corollary 3.9.

\section{Transference of Maximal Estimates by Isometries}

The following theorem uses isometries to transfer to sigma-finite measure spaces the strong type bounds for discrete bisublinear maximal operators.

Theorem 4.1. Suppose that the exponents $p_{1}, p_{2}$, and $p_{3}$ satisfy the conditions:

$$
\begin{gathered}
0<p_{1}, p_{2} \leq \infty \\
\frac{1}{p_{1}}+\frac{1}{p_{2}}=\frac{1}{p_{3}} \\
0<p_{3}<\infty .
\end{gathered}
$$


For each $j \in \mathbb{N}$, let $\Lambda^{(j)} \equiv\left\{\Lambda_{n}^{(j)}\right\}_{n=-\infty}^{\infty}$ be a finitely supported sequence of complex numbers, and suppose that there is a constant $\mathfrak{c}$ such that, in the notation of Definition 1.2, the bisublinear maximal operator $\mathfrak{S}_{\left\{\Lambda^{(j)}\right\}}^{b}$ satisfies

$$
\begin{array}{r}
\left\|\mathfrak{S}_{\left\{\Lambda^{(j)}\right\}}^{b}(a, b)\right\|_{\ell^{p_{3}}(\mathbb{Z})} \leq \mathfrak{c}\|a\|_{\ell^{p_{1}}(\mathbb{Z})}\|b\|_{\ell^{p_{2}}(\mathbb{Z})}, \\
\text { for all } a \in \ell^{p_{1}}, \text { and all } b \in \ell^{p_{2}}(\mathbb{Z}) .
\end{array}
$$

Let $(\Omega, \mu)$ be a sigma-finite measure space, and let $U$ be a bijective linear mapping of $\mathcal{A}(\mu)$ onto $\mathcal{A}(\mu)$ such that the following two conditions hold:

(i) Whenever $\left\{g_{k}\right\}_{k=1}^{\infty} \subseteq \mathcal{A}(\mu), g \in \mathcal{A}(\mu)$, and $g_{k} \rightarrow g \mu$-a.e. on $\Omega$, it follows that as $k \rightarrow \infty, U\left(g_{k}\right) \rightarrow U(g) \mu$-a.e. on $\Omega$, and $U^{-1}\left(g_{k}\right) \rightarrow U^{-1}(g)$ $\mu$-a.e. on $\Omega$.

(ii) For $\nu=1,2,3$, the restriction $\left(U \mid L^{p_{\nu}}(\mu)\right)$ is a surjective linear isometry of $L^{p_{\nu}}(\mu)$.

Define the bisublinear maximal function $\mathfrak{M}$ on $L^{p_{1}}(\mu) \times L^{p_{2}}(\mu)$ by writing for all $f \in L^{p_{1}}(\mu)$, all $g \in L^{p_{2}}(\mu)$, and all $x \in \Omega$,

$$
(\mathfrak{M}(f, g))(x)=\sup _{j \in \mathbb{N}}\left|\sum_{n=-\infty}^{\infty}\left(U^{n} f\right)(x)\left(U^{-n} g\right)(x) \Lambda_{n}^{(j)}\right| .
$$

Then the constant $\mathfrak{c}$ in (4.4) also satisfies

$$
\begin{aligned}
& \|\mathfrak{M}(f, g)\|_{L^{p_{3}}(\mu)} \leq \mathfrak{c}\|f\|_{L^{p_{1}}(\mu)}\|g\|_{L^{p_{2}}(\mu)}, \\
& \quad \text { for all } f \in L^{p_{1}}(\mu), \text { and all } g \in L^{p_{2}}(\mu) .
\end{aligned}
$$

Proof. Since $p_{3}<\infty$, at most one of the exponents $p_{1}, p_{2}$ is $\infty$. It is thus clear from the current hypotheses on the exponents $p_{1}, p_{2}$, and $p_{3}$ that at least two of them are distinct. Consequently the present setup satisfies the hypotheses of Proposition 3.8 and of Corollary 3.9 (whose notation we now follow). Temporarily fix $J \in \mathbb{N}$, and define $\mathfrak{M}^{(J)}$ on $L^{p_{1}}(\mu) \times L^{p_{2}}(\mu)$ by writing for all $f \in L^{p_{1}}(\mu)$, all $g \in L^{p_{2}}(\mu)$, and all $x \in \Omega$,

$$
\left(\mathfrak{M}^{(J)}(f, g)\right)(x)=\sup _{1 \leq j \leq J}\left|\sum_{n=-\infty}^{\infty}\left(U^{n} f\right)(x)\left(U^{-n} g\right)(x) \Lambda_{n}^{(j)}\right| .
$$

Let $f \in L^{p_{1}}(\mu), g \in L^{p_{2}}(\mu)$, and consider the transfomation $V$ of (3.16). It is clear from (3.1), Corollary 3.4, and (3.17) that for each $m \in \mathbb{Z}$, the transformation $V^{m}$ satisfies the following equality $\mu$-a.e. on $\Omega$ :

$$
\begin{aligned}
& \left|\left(V^{m} \mid L^{p_{3}}(\mu)\right)\left(\mathfrak{M}^{(J)}(f, g)\right)\right| \\
& \sup _{1 \leq j \leq J}\left|\sum_{n=-\infty}^{\infty}\left(U^{m+n} f\right)\left(U^{m-n} g\right) \Lambda_{n}^{(j)}\right| .
\end{aligned}
$$


Since $\left(V^{m} \mid L^{p_{3}}(\mu)\right)$ is isometric, we see from (4.7) that the following holds for an arbitrary positive integer $L$.

$$
\begin{aligned}
& (2 L+1)\left\|\mathfrak{M}^{(J)}(f, g)\right\|_{L^{p_{3}(\mu)}}^{p_{3}} \\
= & \sum_{m=-L}^{L}\left\|\sup _{1 \leq j \leq J}\left|\sum_{n=-\infty}^{\infty}\left(U^{m+n} f\right)\left(U^{m-n} g\right) \Lambda_{n}^{(j)}\right|\right\|_{L^{p_{3}(\mu)}}^{p_{3}} \\
= & \int_{\Omega} \sum_{m=-L}^{L} \sup _{1 \leq j \leq J}\left|\sum_{n=-\infty}^{\infty}\left(U^{m+n} f\right)\left(U^{m-n} g\right) \Lambda_{n}^{(j)}\right|^{p_{3}} d \mu .
\end{aligned}
$$

Let $N_{J}$ be the least positive integer such that whenever $1 \leq j \leq J$, and $n \in \mathbb{Z}$ with $|n|>N_{J}$, we have

$$
\Lambda_{n}^{(j)}=0
$$

After denoting by $\xi$ the characteristic function, defined on $\mathbb{Z}$, of $\left\{k \in \mathbb{Z}:|k| \leq L+N_{J}\right\}$, we rewrite $(4.8)$ in the form

$$
\begin{aligned}
& (2 L+1)\left\|\mathfrak{M}^{(J)}(f, g)\right\|_{L^{p_{3}(\mu)}}^{p_{3}} \\
& =\int_{\Omega} \sum_{m=-L}^{L} \sup _{1 \leq j \leq J}\left|\sum_{n=-\infty}^{\infty} \xi(m+n)\left(U^{m+n} f\right) \xi(m-n)\left(U^{m-n} g\right) \Lambda_{n}^{(j)}\right|^{p_{3}} d \mu .
\end{aligned}
$$

We first complete the proof for the case in which both $p_{1}$ and $p_{2}$ are finite. Using the hypothesis in (4.4) to estimate pointwise on $\Omega$ the size of the integrand on the right of (4.9), we find that

$$
\begin{aligned}
& \sum_{m=-L}^{L} \sup _{1 \leq j \leq J}\left|\sum_{n=-\infty}^{\infty} \xi(m+n)\left(U^{m+n} f\right) \xi(m-n)\left(U^{m-n} g\right) \Lambda_{n}^{(j)}\right|^{p_{3}} \\
\leq & \mathfrak{c}^{p_{3}}\left(\sum_{n=-L-N_{J}}^{L+N_{J}}\left|U^{n} f\right|^{p_{1}}\right)^{p_{3} / p_{1}} \cdot\left(\sum_{n=-L-N_{J}}^{L_{+} N_{J}}\left|U^{n} g\right|^{p_{2}}\right)^{p_{3} / p_{2}} \cdot
\end{aligned}
$$

Using this estimate inside the integral on the right of (4.9), we deduce that

$$
\begin{aligned}
& (2 L+1)\left\|\mathfrak{M}^{(J)}(f, g)\right\|_{L^{p_{3}(\mu)}}^{p_{3}} \\
\leq & \mathfrak{c}^{p_{3}} \int_{\Omega}\left(\sum_{n=-L-N_{J}}^{L+N_{J}}\left|U^{n} f\right|^{p_{1}}\right)^{p_{3} / p_{1}}\left(\sum_{j=-L-N_{J}}^{L+N_{J}}\left|U^{n} g\right|^{p_{2}}\right)^{p_{3} / p_{2}} d \mu .
\end{aligned}
$$

After applying Hölder's inequality on the right for the pair of conjugate indices $\left(p_{1} / p_{3}\right)$ and $\left(p_{2} / p_{3}\right)$, and then taking into account that $\left(U \mid L^{p_{1}}(\mu)\right),\left(U \mid L^{p_{2}}(\mu)\right)$ are surjective isometries, we see that

$$
\begin{aligned}
& \left\|\mathfrak{M}^{(J)}(f, g)\right\|_{L^{p_{3}(\mu)}}^{p_{3}} \\
\leq & \frac{2 L+2 N_{J}+1}{2 L+1} \mathfrak{c}^{p_{3}}\|f\|_{L^{p_{1}}(\mu)}^{p_{3}}\|g\|_{L^{p_{2}(\mu)}}^{p_{3}} .
\end{aligned}
$$


While keeping $J$ fixed, we now let $L \rightarrow \infty$ on the right. This shows that for each $J \in \mathbb{N}$, all $f \in L^{p_{1}}(\mu)$, and all $g \in L^{p_{2}}(\mu)$,

$$
\left\|\mathfrak{M}^{(J)}(f, g)\right\|_{L^{p_{3}(\mu)}}^{p_{3}} \leq \mathfrak{c}^{p_{3}}\|f\|_{L^{p_{1}}(\mu)}^{p_{3}}\|g\|_{L^{p_{2}(\mu)}}^{p_{3}} .
$$

We can now let $J \rightarrow \infty$ in (4.11) to obtain (4.6) by monotone convergence.

In the remaining case, precisely one of the exponents $p_{1}, p_{2}$ is $\infty$ (for sake of definiteness, say $p_{2}=\infty$, which implies that $p_{3}=p_{1}$ ), and then by making obvious corresponding adjustments to the reasoning subsequent to (4.9) we arrive at the following substitute estimate for (4.10).

$$
\begin{aligned}
& \left\|\mathfrak{M}^{(J)}(f, g)\right\|_{L^{p_{3}(\mu)}}^{p_{3}} \\
\leq & \frac{2 L+2 N_{J}+1}{2 L+1} \mathfrak{c}^{p_{3}}\|f\|_{L^{p_{1}}(\mu)}^{p_{1}}\left\|\sup _{|n| \leq L+N_{J}}\left|U^{n} g\right|\right\|_{L^{\infty}(\mu)}^{p_{3}} .
\end{aligned}
$$

Since for each $n \in \mathbb{Z},\left(U^{n} \mid L^{\infty}(\mu)\right)$ is isometric, we have pointwise $\mu$-a.e. on $\Omega$,

$$
\left|U^{n} g\right| \leq\left\|U^{n} g\right\|_{L^{\infty}(\mu)}=\|g\|_{L^{\infty}(\mu)},
$$

and so

$$
\begin{aligned}
& \left\|\mathfrak{M}^{(J)}(f, g)\right\|_{L^{p_{3}(\mu)}}^{p_{3}} \\
\leq & \frac{2 L+2 N_{J}+1}{2 L+1} \mathfrak{c}^{p_{3}}\|f\|_{L^{p_{1}(\mu)}}^{p_{1}}\|g\|_{L^{\infty}(\mu)}^{p_{3}} .
\end{aligned}
$$

Since $p_{3}=p_{1}$ in the case at hand, the proof of (4.6) in this case can now be completed as before by first letting $L \rightarrow \infty$ and then letting $J \rightarrow \infty$.

Upon specializing the sequence of discrete kernels $\left\{\Lambda^{(j)}\right\}_{j=1}^{\infty}$ in Theorem 4.1 by writing for each $j \in \mathbb{N}$, and each $n \in \mathbb{Z}$,

$$
\Lambda_{n}^{(j)}=\left\{\begin{array}{cc}
n^{-1}, & \text { if } 0<|n| \leq j \\
0, & \text { otherwise }
\end{array}\right.
$$

we arrive at the following transferred version of the discrete bisublinear maximal Hilbert transform in Theorem 1.4 .

Theorem 4.2. Suppose that the exponents $p_{1}, p_{2}$, and $p_{3}$ satisfy the conditions (1.12), (1.13), and (1.14). Let $(\Omega, \mu)$ be a sigma-finite measure space, and let $U$ be a bijective linear mapping of $\mathcal{A}(\mu)$ onto $\mathcal{A}(\mu)$ such that the conditions (i) and (ii) in Theorem 4.1 hold. Define the bisublinear maximal function $\mathcal{H}_{U}$ on $L^{p_{1}}(\mu) \times L^{p_{2}}(\mu)$ by writing for all $f \in L^{p_{1}}(\mu)$, all $g \in L^{p_{2}}(\mu)$, and all $x \in \Omega$,

$$
\left(\mathcal{H}_{U}(f, g)\right)(x)=\sup _{j \in \mathbb{N}}\left|\sum_{0<|n| \leq j} \frac{\left\{\left(U^{n} f\right)(x)\right\}\left\{\left(U^{-n} g\right)(x)\right\}}{n}\right| .
$$

Then

$$
\begin{aligned}
&\left\|\mathcal{H}_{U}(f, g)\right\|_{L^{p_{3}}(\mu)} \leq \mathfrak{A}_{p_{1}, p_{2}}\|f\|_{L^{p_{1}}(\mu)}\|g\|_{L^{p_{2}}(\mu)}, \\
& \text { for all } f \in L^{p_{1}}(\mu), \text { and all } g \in L^{p_{2}}(\mu),
\end{aligned}
$$

where $\mathfrak{A}_{p_{1}, p_{2}}$ is the constant depending only on $p_{1}$ and $p_{2}$ which appears in (1.22). 
Similarly, after specifying the sequence of discrete kernels $\left\{\Lambda^{(j)}\right\}_{j=1}^{\infty}$ in Theorem 4.1 by writing for each $j \in \mathbb{N}$, and each $n \in \mathbb{Z}$,

$$
\Lambda_{n}^{(j)}=\left\{\begin{array}{cl}
(2 j+1)^{-1}, & \text { if }|n| \leq j \\
0, & \text { otherwise. }
\end{array}\right.
$$

we can obtain the following transferred version of the discrete bisublinear HardyLittlewood maximal operator in Theorem 1.5.

Theorem 4.3. Suppose that the exponents $p_{1}, p_{2}$, and $p_{3}$ satisfy the conditions (1.17) and (1.18). Let $(\Omega, \mu)$ be a sigma-finite measure space, and let $U$ be a bijective linear mapping of $\mathcal{A}(\mu)$ onto $\mathcal{A}(\mu)$ such that the conditions ( $i)$ and (ii) in Theorem 4.1 hold. Define the bisublinear maximal function $\mathcal{M}_{U}$ on $L^{p_{1}}(\mu) \times L^{p_{2}}(\mu)$ by writing for all $f \in L^{p_{1}}(\mu)$, all $g \in L^{p_{2}}(\mu)$, and all $x \in \Omega$,

$$
\left(\mathcal{M}_{U}(f, g)\right)(x)=\sup _{j \in \mathbb{N}} \frac{1}{2 j+1} \sum_{n=-j}^{j}\left|\left(U^{n} f\right)(x)\right|\left|\left(U^{-n} g\right)(x)\right| \text {. }
$$

Then

$$
\begin{aligned}
& \left\|\mathcal{M}_{U}(f, g)\right\|_{L^{p_{3}(\mu)}} \leq \mathfrak{B}_{p_{1}, p_{2}}\|f\|_{L^{p_{1}}(\mu)}\|g\|_{L^{p_{2}(\mu)}}, \\
& \text { for all } f \in L^{p_{1}}(\mu) \text {, and all } g \in L^{p_{2}}(\mu),
\end{aligned}
$$

where $\mathfrak{B}_{p_{1}, p_{2}}$ is the constant depending only on $p_{1}$ and $p_{2}$ which appears in (1.24).

Proof. Since the absolute value signs on the right of (4.12) occur inside the summation sign, the form of $\mathcal{M}_{U}$ does not, strictly speaking, conform to the definition of $\mathfrak{M}$ in Theorem 4.1. This detail is easily attended to, since the automorphism $\Phi_{1}$ of $\mathcal{A}(\mu)$ which Proposition 3.8 associates with $U$ also satisfies the hypotheses of Theorem 4.1 in place of $U$. In order to obtain the present theorem for $\mathcal{M}_{U}$ as defined in (4.12), we need only apply Theorem 4.1 to $\Phi_{1}$ in place of $U$ and to $|f|$ and $|g|$ for arbitrary $f \in L^{p_{1}}(\mu)$ and arbitrary $g \in L^{p_{2}}(\mu)$.

For the transference of multisublinear versions of the Hardy-Littlewood maximal operator to a discrete dynamical system via the system's measure-preserving transformation, see Proposition 14.1 in [14]. In the case of the discrete bisublinear Hardy-Littlewood maximal operator, such transference to dynamical systems automatically furnishes some expansion to the range of exponents for which the a.e. convergence in Bourgain's double recurrence theorem holds, and we now briefly describe this state of affairs. In [8] J. Bourgain established the following double recurrence theorem (see the discussion on pg. 140 of [8] regarding the a.e. convergence for $L^{2}$-functions quoted here).

Theorem 4.4. Suppose that $(\Omega, \mu)$ is a measure space such that $\mu(\Omega)<\infty$, and $T$ is an invertible measure-preserving transformation of $\Omega$ onto $\Omega$. Then for all $f \in L^{2}(\mu)$, and all $g \in L^{2}(\mu)$,

$$
\frac{1}{N} \sum_{n=1}^{N} f\left(T^{n} x\right) \cdot g\left(T^{-n} x\right)
$$

converges for $\mu$-almost all $x \in \Omega$, as $N \rightarrow \infty$.

In view of the Banach Principle in Proposition 3.1 and the strong type boundedness of the discrete bisublinear Hardy-Littlewood maximal operator transferred 
via $T$, it is clear that the almost everywhere convergence of the bilinear ergodic averages in (4.14) will hold whenever $f \in L^{p_{1}}(\mu)$, and $g \in L^{p_{2}}(\mu)$, provided

$$
\begin{gathered}
1<p_{1}, p_{2}<\infty ; \\
\frac{1}{p_{1}}+\frac{1}{p_{2}}<\frac{3}{2} .
\end{gathered}
$$

Example 4.1. Suppose that (4.15) and (4.16) hold. Let $m \in \mathbb{N}$, let $\mu$ be Lebesgue measure of $\mathbb{R}^{m}$, and suppose that $\mathfrak{U}$ is an orthogonal linear transformation of $\mathbb{R}^{m}$ onto $\mathbb{R}^{m}$. Since $|\operatorname{det}(\mathfrak{U})|=1, \mathfrak{U}$ is an invertible measure-preserving transformation of $\left(\mathbb{R}^{m}, \mu\right)$. For each $k \in \mathbb{N}$, denote by $\mathcal{B}_{k}$ the closed $k$-ball $\left\{x \in \mathbb{R}^{m}:\|x\|_{\mathbb{R}^{m}} \leq k\right\}$. Then $\left(\mathfrak{U} \mid \mathcal{B}_{k}\right)$ is an invertible measure-preserving transformation of $\mathcal{B}_{k}$. Since $\mu\left(\mathcal{B}_{k}\right)<$ $\infty$, we can apply the preceding observation to see that if $f \in L^{p_{1}}\left(\mathbb{R}^{m}, \mu\right)$ and $g \in L^{p_{2}}\left(\mathbb{R}^{m}, \mu\right)$, then the bilinear ergodic averages

$$
\frac{1}{N} \sum_{n=1}^{N} f\left(\mathfrak{U}^{n} x\right) \cdot g\left(\mathfrak{U}^{-n} x\right)
$$

converge for $\mu$-almost all $x$ in each $\mathcal{B}_{k}$, and hence converge for $\mu$-almost all $x \in \mathbb{R}^{m}$.

\section{An Application to a.e. Convergence of the Transferred Bilinear Hilbert Averages}

The following theorem applies Theorem 4.2 to infer almost everywhere convergence for the bilinear Hilbert averages induced by translations of locally compact abelian groups.

Theorem 5.1. Let $\lambda$ be Haar measure for a locally compact abelian group $G$, and let $\tau \in G$. Denote by $U_{\tau}$ the translation operator corresponding to $\tau$ that acts on the algebra $\mathcal{A}(\lambda)$, consisting of all complex-valued $\lambda$-measurable functions on $G$ (identified modulo equality $\lambda$-a.e. on $G$ ). Suppose that the exponents $p_{1}, p_{2}$, and $p_{3}$ satisfy the conditions

$$
\begin{gathered}
1<p_{1}, p_{2}<\infty ; \\
\frac{1}{p_{1}}+\frac{1}{p_{2}}=\frac{1}{p_{3}}<\frac{3}{2} .
\end{gathered}
$$

Define the bisublinear mapping $\mathfrak{H}_{G, \tau}$ on $L^{p_{1}}(\lambda) \times L^{p_{2}}(\lambda)$ by writing for all $f \in$ $L^{p_{1}}(\lambda)$, all $g \in L^{p_{2}}(\lambda)$, and all $x \in G$,

$$
\left(\mathfrak{H}_{G, \tau}(f, g)\right)(x)=\sup _{N \in \mathbb{N}}\left|\sum_{0<|n| \leq N} \frac{\left\{\left(U_{\tau}^{n} f\right)(x)\right\}\left\{\left(U_{\tau}^{-n} g\right)(x)\right\}}{n}\right| .
$$

Then for all $f \in L^{p_{1}}(\lambda)$, and all $g \in L^{p_{2}}(\lambda)$, we have:

(i) $\left\|\mathfrak{H}_{G, \tau}(f, g)\right\|_{L^{p_{3}(\lambda)}} \leq \mathfrak{A}_{p_{1}, p_{2}}\|f\|_{L^{p_{1}}(\lambda)}\|g\|_{L^{p_{2}(\lambda)}}$, where $\mathfrak{A}_{p_{1}, p_{2}}$ is the constant depending only on $p_{1}$ and $p_{2}$ that occurs in (1.22);

(ii) the sequence of functions

$$
\left\{\sum_{0<|n| \leq N} \frac{\left(U_{\tau}^{n} f\right) \cdot\left(U_{\tau}^{-n} g\right)}{n}\right\}_{N=1}^{\infty}
$$

converges $\lambda$-a.e. on $G$ to a function $H_{G, \tau}(f, g) \in L^{p_{3}}(\lambda)$ such that

$$
\left\|H_{G, \tau}(f, g)\right\|_{L^{p_{3}}(\lambda)} \leq \mathfrak{A}_{p_{1}, p_{2}}\|f\|_{L^{p_{1}}(\lambda)}\|g\|_{L^{p_{2}}(\lambda)} .
$$


Before taking up the proof of Theorem 5.1, we make a few simplifying observations which, in particular, furnish a reduction to the case of sigma-finite $\lambda$, as required for satisfying the hypotheses of Theorem 4.2. We begin this process by recalling a few items from the structure theory of locally compact groups.

Definition 5.1. A topological group $\mathcal{G}$ is said to be compactly generated provided there is a compact subset $F$ of $\mathcal{G}$ such that $\mathcal{G}$ coincides with the abstract subgroup generated by $F$.

Notice that a compactly generated group is automatically sigma-compact. The following standard item (see, e.g., Theorem (5.14) in [21]) fits compactly generated groups into analysis on locally compact groups.

Proposition 5.2. Let $F$ be a compact subset of a locally compact group $\mathcal{G}$. Then there is an open and closed compactly generated subgroup of $\mathcal{G}$ that contains $F$.

We remark that the existence of an open sigma-compact subgroup of the arbitrary locally compact group $\mathcal{G}$ can be used to show that every subset of $\mathcal{G}$ which has finite left Haar measure is sigma-bounded (that is, is a subset of the union of a sequence of compact sets). The following item from the structure theory of locally compact abelian groups (Theorem (9.8) in [21]) will play a central role in establishing Theorem 5.1 in the full generality stated.

Proposition 5.3. Every compactly generated locally compact abelian group $\mathcal{G}$ is topologically isomorphic with a direct product

$$
\mathbb{R}^{a} \times \mathbb{Z}^{b} \times K
$$

where $a$ and $b$ are non-negative integers, and $K$ is a compact abelian group.

We claim that the proof of Theorem 5.1 can be reduced to the special case in which $G$ is compactly generated. To establish the claim, assume that Theorem 5.1 is known to be valid when $G$ is compactly generated, and consider the general case where $G$ is an arbitrary locally compact abelian group. Let $f \in L^{p_{1}}(\mu), g \in L^{p_{2}}(\mu)$. Then there is a sequence $\left\{F_{k}\right\}_{k=1}^{\infty}$ of compact subsets of $G$ such that

$$
\{t \in G: f(t) \neq 0\} \subseteq \bigcup_{k=1}^{\infty} F_{k} .
$$

Consequently we can apply Proposition 5.2 to obtain an increasing sequence $\left\{G_{k}\right\}_{k=1}^{\infty}$ consisting of open and closed compactly generated subgroups of $G$, and such that for each $k \in \mathbb{N}$,

$$
F_{k} \bigcup\{\tau\} \subseteq G_{k}
$$

(Since each subgroup $G_{k}$ is open, the Haar measure $\lambda$ of $G$, when restricted to $G_{k}$, serves as Haar measure for $G_{k}$.) For each $k \in \mathbb{N}$, we can temporarily confine our attention to $G_{k}$, and there apply the special case of Theorem 5.1 to infer that

$$
\begin{aligned}
& \left\|\sup _{N \in \mathbb{N}}\left|\sum_{0<|n| \leq N} \frac{\left(U_{\tau}^{n} f\right) \cdot\left(U_{\tau}^{-n} g\right)}{n}\right|\right\|_{L^{p_{3}\left(G_{k}, \lambda\right)}} \\
\leq & \mathfrak{A}_{p_{1}, p_{2}}\|f\|_{L^{p_{1}(G, \lambda)}}\|g\|_{L^{p_{2}(G, \lambda)}} .
\end{aligned}
$$

Observe that for all $x \in G \backslash\left(\bigcup_{k=1}^{\infty} G_{k}\right)$, and all $n \in \mathbb{Z}$, we have $\left(U_{\tau}^{n} f\right)(x)=0$. Hence we can let $k \rightarrow \infty$ in (5.7) to deduce by monotone convergence that the assertion in 
Theorem 5.1-(i) holds. The assertion in Theorem 5.1-(ii) can be justified by using the sequence $\left\{G_{k}\right\}_{k=1}^{\infty}$ to argue similarly from the special case of the theorem.

Proof of Theorem 5.1. As has just been described, we can (and will) assume without loss of generality that $G$ is compactly generated. Since, in particular, $G$ is sigma-compact, we can apply Theorem 4.2 directly to $(G, \lambda)$ in order to obtain conclusion (i) of the theorem.

So it now remains only to establish the $\lambda$-a.e. convergence of the bilinear Hilbert averages, as asserted in conclusion (ii) of the theorem. For this purpose we proceed to show that the a.e.-convergence hypothesis of the multilinear Banach Principle (Proposition 3.1) is satisfied here. By Proposition 5.3, we can write $G$ in the form (5.4). Thus, in the sense of integration over locally compact spaces (as treated by Chapter III of [21]), $\lambda$ is the product measure of the Haar measures $\lambda_{1}, \lambda_{2}, \lambda_{3}$ of $\mathbb{R}^{a}, \mathbb{Z}^{b}$, and $K$, respectively. Our method of proof will exhaust the separate cases which arise according to the possible combinations of values for the non-negative integers $a$ and $b$ in (5.4).

Case I $(a=b=0)$. In this case $G$ can be identified with the compact abelian group $K$. Since it follows with the aid of the Stone-Weierstrass Theorem that the trigonometric polynomials on $K$ (that is, the finite linear combinations of continuous characters of $K$ ) are dense in $L^{p}(\lambda)$ for $0<p<\infty$, it suffices for Case I to observe that for all continuous characters $\gamma_{1}, \gamma_{2}$ of $K$, and for every $x \in K$, the sequence

$$
\begin{gathered}
\left\{\sum_{0<|n| \leq N} \frac{\left(\left(U_{\tau}^{n} \gamma_{1}\right)(x)\right)\left(\left(U_{\tau}^{-n} \gamma_{2}\right)(x)\right)}{n}\right\}_{N=1}^{\infty} \\
=\left\{\gamma_{1}(x) \gamma_{2}(x) \sum_{0<|n| \leq N} \frac{\left(\gamma_{1}(\tau) \overline{\gamma_{2}(\tau)}\right)^{n}}{n}\right\}_{N=1}^{\infty}
\end{gathered}
$$

converges. The convergence of the sequence on the right is elementary, since the function $\phi$ of bounded variation on the circle specified by $\phi(1)=0$, and

$$
\phi\left(e^{i t}\right)=i(\pi-t), \text { for } 0<t<2 \pi,
$$

has Fourier series

$$
\sum_{\substack{n=-\infty \\ n \neq 0}}^{\infty} \frac{z^{n}}{n},
$$

which converges to $\phi(z)$ at every $z \in \mathbb{T}$.

Case II $(a=0, b>0)$. In this case $G$ can be identified with the direct product $\mathbb{Z}^{b} \times K$. Put

$$
\tau=(u, v) \in \mathbb{Z}^{b} \times K,
$$

and for $\mathfrak{m} \in \mathbb{Z}^{b}$, let $\eta_{\mathfrak{m}}$ denote the characteristic function, defined on $\mathbb{Z}^{b}$, of the singleton set consisting of $\mathfrak{m}$. For $0<p<\infty$ we can take as a dense subset of $L^{p}(\lambda)$ the linear span of all functions having the form

$$
(x, y) \in \mathbb{Z}^{b} \times K \mapsto \eta_{\mathfrak{m}}(x) \gamma(y),
$$

where $\mathfrak{m} \in \mathbb{Z}^{b}$, and $\gamma$ is a continuous character of $K$. So for Case II it suffices to show that if $\mathfrak{m}_{1} \in \mathbb{Z}^{b}, \mathfrak{m}_{2} \in \mathbb{Z}^{b}, \gamma_{1}$ and $\gamma_{2}$ are continuous characters of $K$, and 
$(x, y) \in \mathbb{Z}^{b} \times K$, then the sequence

$$
\left\{\gamma_{1}(y) \gamma_{2}(y) \sum_{0<|n| \leq N} \frac{\eta_{\mathfrak{m}_{1}}(x+n u) \eta_{\mathfrak{m}_{2}}(x-n u)\left(\gamma_{1}(v) \overline{\gamma_{2}(v)}\right)^{n}}{n}\right\}_{N=1}^{\infty}
$$

is convergent. This convergence can readily be see as follows. If $u=0$, then the convergence is immediate by virtue of the above-noted pointwise convergence on $\mathbb{T}$ of the Fourier series in (5.8). If $u \neq 0$, then $\eta_{\mathfrak{m}_{1}}(x+n u)=0$ as soon as

$$
|n|\|u\|_{\mathbb{Z}^{b}}-\|x\|_{\mathbb{Z}^{b}}>\left\|\mathfrak{m}_{1}\right\|_{\mathbb{Z}^{b}}
$$

and so there is $N_{0} \in \mathbb{N}$ such that the sequence in (5.9) has the same value for all $N \geq N_{0}$.

Case III $(a>0, b=0)$. In this case $G$ can be identified with the direct product $\mathbb{R}^{a} \times K$, and for $0<p<\infty$ we can take as a dense subset of $L^{p}(\lambda)$ the linear span of all functions having the form

$$
(x, y) \in \mathbb{R}^{a} \times K \mapsto \psi(x) \gamma(y),
$$

where $\psi: \mathbb{R}^{a} \rightarrow \mathbb{C}$ is a continuous function with compact support, and $\gamma$ is a continuous character of $K$. The proof for Case III can be carried out in analogy with the proof for Case II by replacing the functions $\eta_{\mathfrak{m}}\left(\mathfrak{m} \in \mathbb{Z}^{b}\right)$ in the reasoning for Case II with the continuous compactly supported functions $\psi$ on $\mathbb{R}^{a}$.

Case IV $(a>0, b>0)$. In this case $G$ is identified with the direct product $\mathbb{R}^{a} \times \mathbb{Z}^{b} \times K$, and the details of the proof are similar to those in the preceding cases.

Remark 5.1. Theorem 5.1 does not have a valid analogue for the one-sided bilinear Hilbert averages induced by a translation operator because of the following fundamental feature of ergodic theory (shown independently in [25] and as Corollary 2 of [23] ).

Example 5.1. Whenever we are given an invertible ergodic measure-preserving transformation $\zeta$ of a nonatomic probability space $(\Omega, \mu)$, there is always an $f_{0} \in$ $L^{\infty}(\mu)$ with $\int_{\Omega} f_{0} d \mu=0$ such that

$$
\sup _{N \in \mathbb{N}}\left|\sum_{n=1}^{N} \frac{f_{0}\left(\zeta^{n}(x)\right)}{n}\right|=\infty, \text { for } \mu \text {-almost all } x \in \Omega .
$$

Example 5.1 obviously extends to the one-sided bilinear Hilbert averages in the ergodic theory setting, since we can let $g_{0} \in L^{\infty}(\mu)$ be the function identically equal to 1 on $\Omega$ in order to conclude that

$$
\sup _{N \in \mathbb{N}}\left|\sum_{n=1}^{N} \frac{f_{0}\left(\zeta^{n}(x)\right) g_{0}\left(\zeta^{-n}(x)\right)}{n}\right|=\infty, \text { for } \mu \text {-almost all } x \in \Omega .
$$

In particular, if we take $\Omega$ to be $\mathbb{T}$ with its normalized Haar measure $\lambda$, we can satisfy the hypotheses of Example 5.1 by choosing an arbitrary irrational number $\mathfrak{s}$, putting $\tau=e^{2 \pi i \mathfrak{s}}$, and then taking translation of $\mathbb{T}$ by $\tau$ as the ergodic transformation $\zeta$. Clearly, the hypotheses of Theorem 5.1 on $G, \lambda$, and $\tau$ are also satisfied under these circumstances, but (5.10) shows that the analogues of Theorem 5.1(i),(ii) for the one-sided bilinear Hilbert averages can fail to hold. 


\section{Transference of Bisublinear Maximal Estimates from lCa Groups}

In this last section, we develop a general approach to the transference of bisublinear maximal estimates from arbitrary locally compact abelian groups to Lebesgue spaces of abstract sigma-finite measures. We begin by introducing the requisite machinery and notation, which will be in effect henceforth. The exponents $p_{1}, p_{2}$, and $p_{3}$ will satisfy

$$
\begin{aligned}
& 1 \leq p_{1}, p_{2}<\infty \\
& \frac{1}{p_{1}}+\frac{1}{p_{2}}=\frac{1}{p_{3}} .
\end{aligned}
$$

$\mathcal{G}$ will be an arbitrary locally compact abelian group with Haar measure $\lambda$. We generalize Definition 1.1 to the setting of $\mathcal{G}$ as follows.

Definition 6.1. For $\mathfrak{K} \in L^{1}(\mathcal{G})$, we shall denote by $B_{\mathfrak{K}}$ the bilinear mapping of $L^{2}(\mathcal{G}) \times L^{2}(\mathcal{G})$ into $L^{1}(\mathcal{G})$ specified by

$$
\left(B_{\mathfrak{K}}(f, g)\right)(x)=\int_{\mathcal{G}} f(x+y) g(x-y) \mathfrak{K}(y) d \lambda(y) .
$$

Given a sequence $\left\{\mathfrak{K}_{j}\right\}_{j=1}^{\infty} \subseteq L^{1}(\mathcal{G})$, the corresponding bisublinear maximal operator will be symbolized by $B_{\left\{\mathfrak{K}_{j}\right\}}^{\mathrm{b}}$. Thus, for $f, g \in L^{2}(\mathcal{G})$, and almost all $x \in \mathcal{G}$,

$$
\left(B_{\left\{\mathfrak{K}_{j}\right\}}^{b}(f, g)\right)(x)=\sup _{j \in \mathbb{N}}\left|\left(B_{\mathfrak{K}_{j}}(f, g)\right)(x)\right| .
$$

We now suppose that we are given a sequence of compactly supported functions $\left\{K_{j}\right\}_{j=1}^{\infty} \subseteq L^{1}(\mathcal{G})$ such that for some constant $\rho$,

$$
\begin{aligned}
& \left\|B_{\left\{K_{j}\right\}}^{\mathrm{b}}(f, g)\right\|_{L^{p_{3}}(\mathcal{G})} \leq \rho\|f\|_{L^{p_{1}}(\mathcal{G})}\|g\|_{L^{p_{2}}(\mathcal{G})}, \\
& \quad \text { for all } f \in L^{p_{1}}(\mathcal{G}) \bigcap L^{2}(\mathcal{G}) \text {, all } g \in L^{p_{2}}(\mathcal{G}) \bigcap L^{2}(\mathcal{G}) .
\end{aligned}
$$

Our goal will be to transfer the maximal estimate in (6.3) to the setting of an arbitrary sigma-finite measure space $(\Omega, \mu)$, and we now seek to formulate suitable properties for operators representing $\mathcal{G}$ to implement such a transference. In keeping with (3.3), it is desirable for such operators to incorporate the separation-preserving property. Moreover, these operators will have to act simultaneously on $L^{p}(\mu)$ spaces for different values of the exponent $p$, while some extra care will be needed, because we shall have to control the $L^{p_{3}}(\mu)$-norms of vector-valued integrals, even in the cases when $p_{3}<1$. For these purposes, we shall utilize $\mathcal{A}(\mu)$, the algebra (under pointwise operations) consisting of all complex-valued $\mu$-measurable functions on $\Omega$ (identified modulo equality $\mu$-a.e. on $\Omega$ ), to "represent" $\mathcal{G}$ by linear bijections of $\mathcal{A}(\mu)$ onto $\mathcal{A}(\mu)$. Specifically, we shall consider a mapping $R: u \mapsto R_{u}$ of $\mathcal{G}$ with the following properties.

(1) $R$ is a homomorphism of $\mathcal{G}$ into the group (under composition) of all linear bijections of $\mathcal{A}(\mu)$ onto $\mathcal{A}(\mu)$.

(2) For each $u \in \mathcal{G}, \lim _{k} R_{u}\left(g_{k}\right)=R_{u}(g) \mu$-a.e. on $\Omega$, whenever $\left\{g_{k}\right\}_{k=1}^{\infty} \subseteq$ $\mathcal{A}(\mu), g \in \mathcal{A}(\mu)$, and $\lim _{k} g_{k}=g \mu$-a.e. on $\Omega$.

(3) For $\nu=1,2,3, L^{p_{\nu}}(\mu)$ is invariant under $\left\{R_{u}: u \in \mathcal{G}\right\}$, and the mapping $u \in \mathcal{G} \mapsto\left(R_{u} \mid L^{p_{\nu}}(\mu)\right)$ is a strongly continuous homomorphism of $\mathcal{G}$ into the 
group of invertible separation-preserving operators belonging to $\mathfrak{B}\left(L^{p_{\nu}}(\mu)\right)$ such that

$$
\mathfrak{s}_{\nu} \equiv \sup \left\{\left\|R_{u} \mid L^{p_{\nu}}(\mu)\right\|_{\mathfrak{B}\left(L^{p_{\nu}}(\mu)\right)}: u \in \mathcal{G}\right\}<\infty .
$$

Before taking up the transference of (6.3), we shall take up some further properties of $R$ that automatically flow from the above assumptions. One sees easily with the aid of Proposition 3.7 and property (2) above that there are unique families $\left\{h_{u}\right\}_{u \in \mathcal{G}}$ and $\left\{\Phi_{u}\right\}_{u \in \mathcal{G}}$ such that for every $u \in \mathcal{G}: h_{u} \in \mathcal{A}(\mu)$ with $\left|h_{u}\right|>0$ on $\Omega ; \Phi_{u}$ is an algebra automorphism of $\mathcal{A}(\mu)$ onto $\mathcal{A}(\mu)$; for all $f \in \mathcal{A}(\mu)$, $R_{u} f=h_{u} \Phi_{u}(f)$; whenever $\left\{g_{k}\right\}_{k=1}^{\infty} \subseteq \mathcal{A}(\mu), g \in \mathcal{A}(\mu)$, and $\lim _{k} g_{k}=g \mu$-a.e. on $\Omega$, it follows that $\lim _{k} \Phi_{u}\left(g_{k}\right)=\Phi_{u}(g) \mu$-a.e. on $\Omega$. It is now straighforward to deduce the following analogues in the present context for (3.11), (3.12), and (3.13).

$$
\begin{aligned}
\left|\Phi_{u}(f)\right|^{\alpha} & =\Phi_{u}\left(|f|^{\alpha}\right), \text { for } u \in \mathcal{G}, f \in \mathcal{A}(\mu), 0<\alpha<\infty . \\
\Phi_{u+v}(f) & =\Phi_{u}\left(\Phi_{u}(f)\right), \text { for } u \in \mathcal{G}, v \in \mathcal{G}, f \in \mathcal{A}(\mu) . \\
h_{u+v} & =h_{u} \Phi_{u}\left(h_{v}\right), \text { for } u \in \mathcal{G}, v \in \mathcal{G} .
\end{aligned}
$$

We next show that, since $p_{3}<p_{1}$, we can infer from (6.4) that

$$
\begin{aligned}
\left(\mathfrak{s}_{1}^{p_{1}} \mathfrak{s}_{3}^{p_{3}}\right)^{-1 /\left(p_{1}-p_{3}\right)} \leq & \left|h_{u}\right| \leq\left(\mathfrak{s}_{1}^{p_{1}} \mathfrak{s}_{3}^{p_{3}}\right)^{1 /\left(p_{1}-p_{3}\right)} \quad \mu \text {-a.e. on } \Omega, \\
& \text { for every } u \in \mathcal{G} .
\end{aligned}
$$

To this end, note first that if $\mu(E)<\infty$, and $u \in \mathcal{G}$, then we have for $v=1,3$,

$$
\mathfrak{s}_{\nu}^{-p_{\nu}} \int_{\Omega}\left|h_{u}\right|^{p_{\nu}} \Phi_{u}\left(\chi_{E}\right) d \mu \leq \mu(E) \leq \mathfrak{s}_{\nu}^{p_{\nu}} \int_{\Omega}\left|h_{u}\right|^{p_{\nu}} \Phi_{u}\left(\chi_{E}\right) d \mu<\infty .
$$

This, together with sigma-finiteness, implies that for every $\mu$-measurable set $W$, each $u \in \mathcal{G}$, and for $\left(\nu_{1}, \nu_{2}\right)=(1,3)$ or $(3,1)$,

$$
\mathfrak{s}_{\nu_{1}}^{-p_{\nu_{1}}} \int_{W}\left|h_{u}\right|^{p_{\nu_{1}}} d \mu \leq \mathfrak{s}_{\nu_{2}}^{p_{\nu_{2}}} \int_{W}\left|h_{u}\right|^{p_{\nu_{2}}} d \mu
$$

Suppose again that $\mu(E)<\infty$, and let $\chi_{B} \in \mathcal{A}(\mu)$, with $\Phi_{u}\left(\chi_{E}\right)=\chi_{B}$. Put

$$
B_{0}=\left\{x \in B: \mathfrak{s}_{\nu_{1}}^{-p_{\nu_{1}}}\left|h_{u}\right|^{p_{\nu_{1}}}>\mathfrak{s}_{\nu_{2}}^{p_{\nu_{2}}}\left|h_{u}\right|^{p_{\nu_{2}}}\right\}
$$

Then

$$
\infty>\mathfrak{s}_{\nu_{1}}^{-p_{\nu_{1}}} \int_{\Omega}\left|h_{u}\right|^{p_{\nu_{1}}} \Phi_{u}\left(\chi_{E}\right) d \mu \geq \mathfrak{s}_{\nu_{1}}^{-p_{\nu_{1}}} \int_{B_{0}}\left|h_{u}\right|^{p_{\nu_{1}}} d \mu \geq \mathfrak{s}_{\nu_{2}}^{p_{\nu_{2}}} \int_{B_{0}}\left|h_{u}\right|^{p_{\nu_{2}}} d \mu .
$$

In view of this and (6.9), we have

$$
\infty>\mathfrak{s}_{\nu_{1}}^{-p_{\nu_{1}}} \int_{B_{0}}\left|h_{u}\right|^{p_{\nu_{1}}} d \mu=\mathfrak{s}_{\nu_{2}}^{p_{\nu_{2}}} \int_{B_{0}}\left|h_{u}\right|^{p_{\nu_{2}}} d \mu
$$

which, together with the definition of $B_{0}$, shows that $\mu$-a.e. in $B_{0}$, we have $\mathfrak{s}_{\nu_{1}}^{-p_{\nu_{1}}}\left|h_{u}\right|^{p_{\nu_{1}}}=\mathfrak{s}_{\nu_{2}}^{p_{\nu_{2}}}\left|h_{u}\right|^{p_{\nu_{2}}}$. And it is now clear from the definition of $B_{0}$ that $\mu\left(B_{0}\right)=0$. Since $(\Omega, \mu)$ is sigma-finite, and $\nu_{1}, \nu_{2}$ are distinct, this yields $(6.8)$.

We next show that for $0<p<\infty, L^{p}(\mu)$ is invariant under $\left\{R_{u}: u \in \mathcal{G}\right\}$, and the mapping $u \in \mathcal{G} \mapsto\left(R_{u} \mid L^{p}(\mu)\right)$ is a homomorphism of $\mathcal{G}$ into the group of invertible separation-preserving operators belonging to $\mathfrak{B}\left(L^{p_{\nu}}(\mu)\right)$ such that

$$
\Theta_{p} \equiv \sup \left\{\left\|R_{u} \mid L^{p}(\mu)\right\|_{\mathfrak{B}\left(L^{p}(\mu)\right)}: u \in \mathcal{G}\right\}<\infty \text {. }
$$


To see this, suppose that $0<p<\infty$, and $f$ is a $\mu$-integrable simple function. Then there is a $\mu$-integrable simple function $g \geq 0$ such that

$$
|f|=g^{p_{1} / p}
$$

and, with the aid of two successive applications of (6.8), we find that for each $u \in \mathcal{G}$,

$$
\begin{aligned}
& \int_{\Omega}\left|R_{u} f\right|^{p} d \mu \\
= & \int_{\Omega}\left|h_{u}\right|^{p} \Phi_{u}\left(g^{p_{1}}\right) d \mu \\
\leq & \left(\mathfrak{s}_{1}^{p_{1}} \mathfrak{s}_{3}^{p_{3}}\right)^{p /\left(p_{1}-p_{3}\right)} \int_{\Omega} \Phi_{u}\left(g^{p_{1}}\right) d \mu \\
\leq & \left(\mathfrak{s}_{1}^{p_{1}} \mathfrak{s}_{3}^{p_{3}}\right)^{p /\left(p_{1}-p_{3}\right)}\left(\mathfrak{s}_{1}^{p_{1}} \mathfrak{s}_{3}^{p_{3}}\right)^{p_{1} /\left(p_{1}-p_{3}\right)} \int_{\Omega}\left|h_{u}\right|^{p_{1}} \Phi_{u}\left(g^{p_{1}}\right) d \mu \\
= & \left(\mathfrak{s}_{1}^{p_{1}} \mathfrak{s}_{3}^{p_{3}}\right)^{p /\left(p_{1}-p_{3}\right)}\left(\mathfrak{s}_{1}^{p_{1}} \mathfrak{s}_{3}^{p_{3}}\right)^{p_{1} /\left(p_{1}-p_{3}\right)}\left\|R_{u} g\right\|_{L^{p_{1}}(\mu)}^{p_{1}} .
\end{aligned}
$$

Since $\left\|R_{u} g\right\|_{L^{p_{1}(\mu)}}^{p_{1}} \leq \mathfrak{s}_{1}^{p_{1}}\|g\|_{L^{p_{1}(\mu)}}^{p_{1}}=\mathfrak{s}_{1}^{p_{1}}\|f\|_{L^{p}(\mu)}^{p}$, we can now use property (2) of $R$ together with Fatou's Lemma to obtain (6.10). At this juncture, the following proposition (Proposition (3.2) of [2]) permits us to infer from (6.1) in conjunction with the strong continuity provision in property (3) above and (6.10) that

for $1 \leq p<\infty$, the representation $u \in \mathcal{G} \mapsto R_{u} \mid L^{p}(\mu)$ is strongly continuous.

Proposition 6.1. Let $G$ be a locally compact abelian group and $(X, \sigma)$ an arbitrary measure space. Suppose that $1 \leq r_{1}, r_{2}<\infty$, and, for $j=1,2$, let $u \mapsto \mathfrak{R}_{j, u}$ be a representation of $G$ in $L^{r_{j}}(\sigma)$ such that

$$
\sup \left\{\left\|\Re_{j, u}\right\|_{\mathfrak{B}\left(L^{r_{j}}(\sigma)\right)}: u \in G\right\}<\infty .
$$

Suppose also that

$$
\mathfrak{R}_{1, u} f=\mathfrak{R}_{2, u} f, \text { for } u \in G \text { and } f \in L^{r_{1}}(\sigma) \bigcap L^{r_{2}}(\sigma) .
$$

Then the representation $u \mapsto \mathfrak{R}_{1, u}$ is strongly continuous if and only if the representation $u \mapsto \mathfrak{R}_{2, u}$ is strongly continuous.

For $0<p<\infty$, we shall, as a convenient abbreviation, denote by $R^{(p)}: u \mapsto R_{u}^{(p)}$ the representation $u \mapsto R_{u} \mid L^{p}(\mu)$ of $\mathcal{G}$. A bonus stemming from (6.10) and (6.11) is that for $f \in L^{2}(\mu)$ and $g \in L^{2}(\mu)$, the pointwise product $\left(R_{u}^{(2)} f\right)\left(R_{-u}^{(2)} g\right)$ moves continuously in $L^{1}(\mu)$ (as a function of $u \in \mathcal{G}$ ) with respect to the norm topology of $L^{1}(\mu)$, and is bounded in $L^{1}(\mu)$-norm. Consequently we can use $L^{1}(\mu)$-valued Bochner integration to formulate the following transference analogue of Definition 6.1 .

Definition 6.2. For $\mathfrak{K} \in L^{1}(\mathcal{G})$, we shall denote by $T_{\mathfrak{K}}$ the bilinear mapping of $L^{2}(\mu) \times L^{2}(\mu)$ into $L^{1}(\mu)$ specified by $L^{1}(\mu)$-valued Bochner integration as follows.

$$
\begin{array}{r}
T_{\mathfrak{K}}(f, g)=\int_{\mathcal{G}}\left(R_{u}^{(2)} f\right)\left(R_{-u}^{(2)} g\right) \mathfrak{K}(u) d \lambda(u), \\
\text { for all } f \in L^{2}(\mu) \text { and all } g \in L^{2}(\mu) .
\end{array}
$$


Given a sequence $\left\{\mathfrak{K}_{j}\right\}_{j=1}^{\infty} \subseteq L^{1}(\mathcal{G})$, we define the corresponding transferred bisublinear maximal operator $T_{\left\{\mathfrak{K}_{j}\right\}}^{b}$ by writing for all $f \in L^{2}(\mathcal{G})$, all $g \in L^{2}(\mathcal{G})$, and almost all $x \in \mathcal{G}$,

$$
\left(T_{\left\{\mathfrak{K}_{j}\right\}}^{b}(f, g)\right)(x)=\sup _{j \in \mathbb{N}}\left|\left(T_{\mathfrak{K}_{j}}(f, g)\right)(x)\right| .
$$

We shall also require an auxiliary representation of $\mathcal{G}$ by bijective linear mappings of $\mathcal{A}(\mu)$ onto $\mathcal{A}(\mu)$ that is designed to furnish suitable muliplicativity properties in analogy with (3.17). This is accomplished analogously as follows.

Lemma 6.2. For each $u \in \mathcal{G}$, let $W_{u}$ be the linear mapping of $\mathcal{A}(\mu)$ into $\mathcal{A}(\mu)$ defined by writing pointwise $\mu$-a.e. on $\Omega$,

$$
W_{u} f=h_{u} R_{u} f=h_{u}^{2} \Phi_{u}(f), \text { for all } f \in \mathcal{A}(\mu) .
$$

Then the mapping $W: u \in \mathcal{G} \mapsto W_{u}$ is a homomorphism of $\mathcal{G}$ into the group (under composition) of all linear bijections of $\mathcal{A}(\mu)$ onto $\mathcal{A}(\mu)$. For $0<p<\infty, L^{p}(\mu)$ is invariant under $\left\{W_{u}: u \in \mathcal{G}\right\}$, and the mapping $u \in \mathcal{G} \mapsto\left(W_{u} \mid L^{p}(\mu)\right)$ is a homomorphism of $\mathcal{G}$ into the group of invertible separation-preserving operators belonging to $\mathfrak{B}\left(L^{p}(\mu)\right)$ such that

$$
\Delta_{p} \equiv \sup \left\{\left\|W_{u} \mid L^{p}(\mu)\right\|_{\mathcal{B}\left(L^{p}(\mu)\right)}: u \in \mathcal{G}\right\}<\infty .
$$

For all $u \in \mathcal{G}, v \in \mathcal{G}, w \in \mathcal{G}$, all $f \in \mathcal{A}(\mu)$, and all $g \in \mathcal{A}(\mu)$, we have, pointwise $\mu$-a.e. on $\Omega$,

$$
W_{u}\left(\left(R_{v} f\right)\left(R_{w} g\right)\right)=\left(R_{u+v} f\right)\left(R_{u+w} g\right) .
$$

Proof. All the assertions follow directly from the corresponding properties of $R$ and (6.8) in conjunction with (6.6) and (6.7).

Before taking up the transference theorem for (6.3), there is one remaining matter that needs to be addressed. This concerns the measure-theoretic technicalities connected with the use of Fubini's theorem that inevitably arise in such transference environments. Specifically, for a given $f \in L^{p}(\mu)$ there is no a priori way to represent an expression such as $\left(R_{u}^{(p)} f\right)(x)$ as a $\lambda \times \mu$ measurable function of $(u, x) \in \mathcal{G} \times \Omega$. A general approach to surmounting such difficulties is furnished by Lemma (2.5) of [5], whose proof supplies the following specialized tool for our present situation. (Here and henceforth, given subsets $A$ and $B$ of $\mathcal{G}$, we shall denote by $A-B$ the group -theoretic difference set consisting of all differences $a-b$ such that $a \in A$ and $b \in B$.)

Proposition 6.3. Let $\mathcal{C}$ be a compact subset of $\mathcal{G}$, let $V$ be an open subset of $\mathcal{G}$ having compact closure, and let $f \in L^{2}(\mu)$. Then there exists a complex-valued $\lambda \times \mu$ measurable function $F$ on $\mathcal{G} \times \Omega$ such that:

(a) $F$ vanishes off $(V-\mathcal{C}) \times \Omega$;

(b) for $\lambda$-almost all $u \in V-\mathcal{C}, F(u, \cdot)$ is a representing function for the equivalence class (modulo equality $\mu$-a.e. on $\Omega$ ) of $R_{u}^{(2)} f$.

The stage is now set for the transference of the maximal estimate in (6.3). This takes the following form.

Theorem 6.4. Let $p_{1}, p_{2}, p_{3}, \mathcal{G}$, and $\lambda$ be as just described above. Suppose that we are given a sequence of compactly supported functions $\left\{K_{j}\right\}_{j=1}^{\infty} \subseteq L^{1}(\mathcal{G})$ such that for some constant $\rho$, (6.3) holds. If $(\Omega, \mu)$ is a sigma-finite measure space, and 
$u \mapsto R_{u}$ is a mapping of $\mathcal{G}$ having the properties (1), (2), and (3) listed above, then, in the notation of Definition 6.2, we have:

$$
\begin{aligned}
& \left\|T_{\left\{K_{j}\right\}}^{b}(f, g)\right\|_{L^{p_{3}(\mu)}} \leq \rho \mathfrak{s}_{1} \mathfrak{s}_{2} \Delta_{p_{3}}\|f\|_{L^{p_{1}(\mu)}}\|g\|_{L^{p_{2}(\mu)}}, \\
& \quad \text { for all } f \in L^{p_{1}}(\mu) \bigcap L^{2}(\mu), \text { and all } g \in L^{p_{2}}(\mu) \bigcap L^{2}(\mu),
\end{aligned}
$$

where $\Delta_{p_{3}}$ is defined by (6.12), and $\mathfrak{s}_{1}$, and $\mathfrak{s}_{2}$ are the constants given by (6.4).

Proof. Let $N \in \mathbb{N}$, and define the truncated maximal operator $T^{(N)}$ by writing for all $f \in L^{2}(\mu), g \in L^{2}(\mu)$,

$$
T^{(N)}(f, g)=\sup _{1 \leq j \leq N}\left|T_{K_{j}}(f, g)\right| .
$$

By the monotone convergence theorem it clearly suffices to show that for all $f \in$ $L^{p_{1}}(\mu) \bigcap L^{2}(\mu)$ and all $g \in L^{p_{2}}(\mu) \bigcap L^{2}(\mu)$,

$$
\left\|T^{(N)}(f, g)\right\|_{L^{p_{3}(\mu)}} \leq \rho \mathfrak{s}_{1} \mathfrak{s}_{2} \Delta_{p_{3}}\|f\|_{L^{p_{1}}(\mu)}\|g\|_{L^{p_{2}(\mu)}} .
$$

To this end, fix $f \in L^{p_{1}}(\mu) \bigcap L^{2}(\mu), g \in L^{p_{2}}(\mu) \bigcap L^{2}(\mu)$, and let $\mathcal{C}$ be a symmetric compact subset of $\mathcal{G}$ that contains the support of $K_{j}$ for $1 \leq j \leq N$. Let $\varepsilon$ be a positive real number. By Lemma (31.36) of [22] (a form of Leptin's condition), we can choose a non-void symmetric open set $V$ in $\mathcal{G}$ such that the closure of $V$ is compact, and

$$
\frac{\lambda(V-\mathcal{C})}{\lambda(V)}<1+\varepsilon
$$

Let $v \in V$. We now apply (3.3) to $T^{(N)}(f, g)$ and to the separation-preserving operator $W_{v} \mid L^{1}(\mu) \in \mathfrak{B}\left(L^{1}(\mu)\right)$, which is described by Lemma 6.2. This gives

$$
\left|\left(W_{v} \mid L^{1}(\mu)\right)\left(T^{(N)}(f, g)\right)\right|=\sup _{1 \leq j \leq N}\left|\left(W_{v} \mid L^{1}(\mu)\right)\left(T_{K_{j}}(f, g)\right)\right| .
$$

For $1 \leq j \leq N$, we see by (6.13) that, in terms of $L^{1}(\mu)$-valued Bochner integration,

$$
\left(W_{v} \mid L^{1}(\mu)\right)\left(T_{K_{j}}(f, g)\right)=\int_{\mathcal{C}}\left(R_{v+u}^{(2)} f\right)\left(R_{v-u}^{(2)} g\right) K_{j}(u) d \lambda(u) .
$$

At this juncture, we observe that by virtue of Proposition 6.3, we can and shall arrange matters so that, without loss of generality, we can regard each of the expressions $\left(R_{u}^{(2)} f\right)(x)$ and $\left(R_{-u}^{(2)} g\right)(x)$ as if it were a jointly measurable function of $(u, x)$ on $(V-\mathcal{C}) \times \Omega$. Moreover, we can treat each of the expressions $\left(R_{s+u}^{(2)} f\right)(x)$ and $\left(R_{s-u}^{(2)} g\right)(x)$ as if it were a jointly measurable function of $(s, u, x)$ on $V \times \mathcal{C} \times \Omega$, whence for $v \in V$, and $1 \leq j \leq N$,

$$
\left(\left(W_{v} \mid L^{1}(\mu)\right)\left(T_{K_{j}}(f, g)\right)\right)(x)=\int_{\mathcal{C}}\left(R_{v+u}^{(2)} f\right)(x)\left(R_{v-u}^{(2)} g\right)(x) K_{j}(u) d \lambda(u),
$$

for $\mu$-almost all $x \in \Omega$.

Although we do not yet know that $T^{(N)}(f, g) \in L^{p_{3}}(\mu)$, it is clear from the invariance provision of Lemma 6.2 that for any $h \in \mathcal{A}(\mu)$, and any $g \in \mathcal{G}$, we have $W_{g} h \in L^{p_{3}}(\mu)$ if and only if $h \in L^{p_{3}}(\mu)$. Keeping this in mind, we will now consider 
expressions such as $\left\|T^{(N)}(f, g)\right\|_{L^{p_{3}(\mu)}}^{p_{3}}$ (which, as far as we know at this juncture, might be infinite). From (6.12) and (6.17) we see that for $v \in V$,

$$
\begin{aligned}
\left\|T^{(N)}(f, g)\right\|_{L^{p_{3}(\mu)}}^{p_{3}} & \leq \Delta_{p_{3}}^{p_{3}}\left\|\left(W_{v} \mid L^{1}(\mu)\right)\left(T^{(N)}(f, g)\right)\right\|_{L^{p_{3}(\mu)}}^{p_{3}} \\
& =\Delta_{p_{3}}^{p_{3}}\left\|\sup _{1 \leq j \leq N}\left|\left(W_{v} \mid L^{1}(\mu)\right)\left(T_{K_{j}}(f, g)\right)\right|\right\|_{L^{p_{3}}(\mu)}^{p_{3}} .
\end{aligned}
$$

Substituting (6.19) in the last member, we get

$$
\begin{aligned}
& \left\|T^{(N)}(f, g)\right\|_{L^{p_{3}(\mu)}}^{p_{3}} \\
\leq & \Delta_{p_{3}}^{p_{3}} \int_{\Omega} \sup _{1 \leq j \leq N}\left|\int_{\mathcal{C}}\left(R_{v+u}^{(2)} f\right)(x)\left(R_{v-u}^{(2)} g\right)(x) K_{j}(u) d \lambda(u)\right|^{p_{3}} d \mu(x) .
\end{aligned}
$$

Averaging this estimate over $V$ with respect to $d \lambda(v)$, and using Fubini's theorem to interchange the order of integration, we find that

$$
\begin{aligned}
& \left\|T^{(N)}(f, g)\right\|_{L^{p_{3}(\mu)}}^{p_{3}} \\
\leq & \frac{\Delta_{p_{3}}^{p_{3}}}{\lambda(V)} \int_{\Omega} \int_{V} \sup _{1 \leq j \leq N}\left|\int_{\mathcal{C}}\left(R_{v+u}^{(2)} f\right)(x)\left(R_{v-u}^{(2)} g\right)(x) K_{j}(u) d \lambda(u)\right|^{p_{3}} d \lambda(v) d \mu(x) .
\end{aligned}
$$

Let $\chi$ denote the characteristic function, defined on $\mathcal{G}$, of $V-\mathcal{C}=V+\mathcal{C}$. In terms of $\chi$ we have the following pointwise estimate on $\Omega$ for the integrand with respect to $d \mu(x)$.

$$
\begin{aligned}
& \int_{V} \sup _{1 \leq j \leq N}\left|\int_{\mathcal{C}}\left(R_{v+u}^{(2)} f\right)(x)\left(R_{v-u}^{(2)} g\right)(x) K_{j}(u) d \lambda(u)\right|^{p_{3}} d \lambda(v) \\
\leq & \int_{V} \sup _{1 \leq j \leq N}\left|\int_{\mathcal{G}} \chi(v+u)\left(R_{v+u}^{(2)} f\right)(x) \chi(v-u)\left(R_{v-u}^{(2)} g\right)(x) K_{j}(u) d \lambda(u)\right|^{p_{3}} d \lambda(v) .
\end{aligned}
$$

Applying the hypothesis (6.3) to the majorant in (6.21), we infer that for $\mu$-almost all $x \in \Omega$,

$$
\begin{aligned}
& \int_{V} \sup _{1 \leq j \leq N}\left|\int_{\mathcal{C}}\left(R_{v+u}^{(2)} f\right)(x)\left(R_{v-u}^{(2)} g\right)(x) K_{j}(u) d \lambda(u)\right|^{p_{3}} d \lambda(v) \\
\leq & \rho^{p_{3}}\left(\int_{V-\mathcal{C}}\left|\left(R_{u} f\right)(x)\right|^{p_{1}} d \lambda(u)\right)^{p_{3} / p_{1}}\left(\int_{V-\mathcal{C}}\left|\left(R_{u} g\right)(x)\right|^{p_{2}} d \lambda(u)\right)^{p_{3} / p_{2}} .
\end{aligned}
$$

Using this estimate in (6.20) gives

$$
\begin{aligned}
& \left\|T^{(N)}(f, g)\right\|_{L^{p_{3}(\mu)}}^{p_{3}} \\
\leq & \frac{\Delta_{p_{3}}^{p_{3}} \rho^{p_{3}}}{\lambda(V)} \int_{\Omega}\left(\int_{V-\mathcal{C}}\left|\left(R_{u} f\right)(x)\right|^{p_{1}} d \lambda(u)\right)^{p_{3} / p_{1}}\left(\int_{V-\mathcal{C}}\left|\left(R_{u} g\right)(x)\right|^{p_{2}} d \lambda(u)\right)^{p_{3} / p_{2}} d \mu(x) .
\end{aligned}
$$


Applying Hölder's inequality for the pair of conjugate exponents $\frac{p_{1}}{p_{3}}$ and $\frac{p_{2}}{p_{3}}$, we see that $\left\|T^{(N)}(f, g)\right\|_{L^{p_{3}(\mu)}}^{p_{3}}$ does not exceed the product of

$$
\frac{\Delta_{p_{3}}^{p_{3}} \rho^{p_{3}}}{\lambda(V)}\left(\int_{\Omega} \int_{V-\mathcal{C}}\left|\left(R_{u} f\right)(x)\right|^{p_{1}} d \lambda(u) d \mu(x)\right)^{p_{3} / p_{1}}
$$

with

$$
\left(\int_{\Omega} \int_{V-\mathcal{C}}\left|\left(R_{u} g\right)(x)\right|^{p_{2}} d \lambda(u) d \mu(x)\right)^{p_{3} / p_{2}}
$$

Hence

$$
\begin{aligned}
& \left\|T^{(N)}(f, g)\right\|_{L^{p_{3}(\mu)}}^{p_{3}} \\
\leq & \rho^{p_{3}} \mathfrak{s}_{1}^{p_{3}} \mathfrak{s}_{2}^{p_{3}} \Delta_{p_{3}}^{p_{3}} \frac{\lambda(V-\mathcal{C})}{\lambda(V)}\|f\|_{L^{p_{1}}(\mu)}^{p_{3}}\|g\|_{L^{p_{2}(\mu)}}^{p_{3}} \\
\leq & \rho^{p_{3}} \mathfrak{s}_{1}^{p_{3}} \mathfrak{s}_{2}^{p_{3}} \Delta_{p_{3}}^{p_{3}}(1+\varepsilon)\|f\|_{L^{p_{1}(\mu)}}^{p_{3}}\|g\|_{L^{p_{2}(\mu)}}^{p_{3}} .
\end{aligned}
$$

Letting $\varepsilon \rightarrow 0$ yields (6.15) and completes the proof of Theorem 6.4.

\section{REFERENCES}

[1] N. Asmar, E. Berkson, and T.A. Gillespie, Transference of strong type maximal inequalities by separation-preserving representations, Amer. J.Math., 113(1991), 47-74

[2] N. Asmar, E. Berkson, and T.A. Gillespie, Transference of weak type maximal inequalities by distributionally bounded representations, Quart. J. Math. Oxford (2), 43(1992), 259-282.

[3] E. Berkson and T.A. Gillespie, Mean-boundedness and Littlewood-Paley for separationpreserving operators, Trans. Amer.Math. Soc., 349(1997), 1169-1189.

[4] E. Berkson, T.A. Gillespie, and P.S. Muhly, Analyticity and spectral decompositions of $L^{p}$ for compact abelian groups, Pacific J. Math.127(1987), 247-260.

[5] E. Berkson, T.A. Gillespie, and P.S. Muhly, Generalized analyticity in UMD spaces, Arkiv för Matematik, 27(1989), 1-14.

[6] E. Berkson, M. Paluszyński, and G. Weiss, Transference couples and their applications to convolution operators and maximal operators, in "Interaction between Functional Analysis, Harmonic Analysis, and Probability", Proceedings of Conference at U. of Missouri-Columbia (May 29-June 3, 1994), Lecture Notes in Pure and Applied Math., vol. 175, Marcel Dekker, Inc., New York, 1996, pp. 69-84.

[7] O. Blasco, M. Carro, and T.A. Gillespie, Bilinear Hilbert transform on measure spaces, J. Fourier Analysis and Appl., 11(2005), 459-470.

[8] J. Bourgain, Double recurrence and almost sure convergence, J. reine angew. Math., 404(1990), 140-161.

[9] A.P. Calderón, Ergodic theory and translation-invariant operators, Proc. Nat. Acad. Sci. U.S.A., 59(1968), 349-353.

[10] R.R. Coifman and G. Weiss, Operators associated with representations of amenable groups, singular integrals induced by ergodic flows, the rotation method and multipliers, Studia Math., 47(1973), 285-303.

[11] R.R. Coifman and G. Weiss, Transference Methods in Analysis, Regional Conf. Series in Math., No. 31, Amer. Math. Soc., Providence, 1977.

[12] J.B. Conway, A Course in Functional Analysis,2nd Edition, Graduate Texts in Math. 96, Springer-Verlag, New York, 1990.

[13] M. de Guzmán, Real Variable Methods in Fourier Analysis, North-Holland Mathematics Studies 46=Notas de Matemática (75), North-Holland Publ., New York, 1981.

[14] C. Demeter, T. Tao, and C. Thiele, Maximal multilinear operators, submitted for publication.

[15] J.L. Doob, Stochastic Processes, Wiley and Sons, New York, 1953.

[16] N. Dunford and J.T. Schwartz, Linear Operators. Part I: General Theory, Pure Appl. Math. 7, Interscience, New York, 1958. 
[17] L. Grafakos and G. Weiss, Transference of multilinear operators, Illinois J. Math., 40(1996), 344-351.

[18] L. Grafakos and P. Honzík, Maximal transference and summability of multilinear Fourier series, J. Australian Math. Soc., 80(2006), 65-80.

[19] H. Helson, Analyticity on compact abelian groups, Algebras in Analysis, Proceedings of 1973 Birmingham Conference-NATO Advanced Study Institute, Academic Press, London, 1975, $1-62$.

[20] H. Helson and J.-P. Kahane, Compact groups with ordered duals. III, J. London Math. Soc. (2), 4(1971/72), 573-575.

[21] E. Hewitt and K. A. Ross, Abstract Harmonic Analysis I, Grundlehren der Math. Wissenschaften (Band 115), Academic Press, New York, 1963.

[22] E. Hewitt and K. A. Ross, Abstract Harmonic Analysis II, Grundlehren der Math. Wissenschaften (Band 152), Springer-Verlag, Berlin, 1970.

[23] S. Kakutani and K. Petersen, The speed of convergence in the ergodic theorem, Monatshefte für Math., 91(1981), 11-18.

[24] C.-H. Kan, Ergodic properties of Lamperti operators, Canadian J. Math., 30(1978), 12061214.

[25] K. Krzyżewski, A remark on the ergodic theorem, Ann. Soc.Math. Pol. Comm.[currently named Comment. Math. Prace Mat.], 21(1980), 137-140.

[26] M. Lacey, The bilinear maximal functions map into $L^{p}$ for $\frac{2}{3}<p \leq 1$, Annals of Math., 151(2000), 35-57.

[27] M. Lacey and C. Thiele, $L^{p}$ estimates on the bilinear Hilbert transform for $2<p<\infty$, Annals of Math., 146(1997), 693-724.

[28] M. Lacey and C. Thiele, On Calderón's conjecture, Annals of Math., 149(1999), 475-496.

[29] J. Lamperti, On the isometries of certain function-spaces, Pacific J. Math., 8(1958), 459-466.

[30] C. Thiele, Time-frequency analysis in the discrete phase plane, Ph.D. thesis, Yale University, 1995.

[31] C. Thiele, The quartile operator and pointwise convergence of Walsh series, Trans. Amer. Math. Soc., 352(2000), 5745-5766.

[32] C. Thiele, The maximal quartile operator, Rev. Mat. Iberoamericana, 17(2001), 107-135.

E. Berkson: Department of Mathematics, University of Illinois, 1409 W. Green St., URBANA, IL 61801 U.S.A.

E-mail address: berkson@math.uiuc.edu

Oscar Blasco: Departamento de Análisis Matemático, Universidad de Valencia, 46100 (Burjassot) Valencia, Spain

E-mail address: Oscar.Blasco@uv.es

M.J. Carro: Dept. App. Math. \& Analysis, Universitat de Barcelona, Gran Via 585, 08071 Barcelona, Spain

E-mail address: carro@ub.edu

T.A. Gillespie: Department of Mathematics and Statistics, University of Edinburgh, James Clerk Maxwell Building, Edinburgh EH9 3JZ, Scotland, UK

E-mail address: alastair@maths.ed.ac.uk 